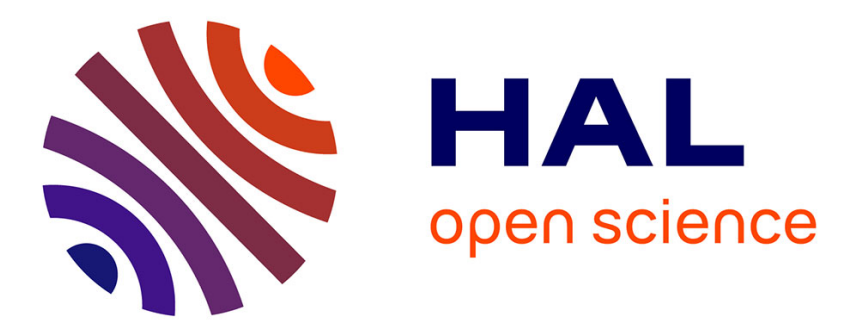

\title{
A new efficient momentum preserving Level-Set/VOF method for high density and momentum ratio incompressible two-phase flows
}

Davide Zuzio, Annagrazia Orazzo, Jean-Luc Estivalèzes, Isabelle Lagrange

\section{- To cite this version:}

Davide Zuzio, Annagrazia Orazzo, Jean-Luc Estivalèzes, Isabelle Lagrange. A new efficient momentum preserving Level-Set/VOF method for high density and momentum ratio incompressible two-phase flows. Journal of Computational Physics, 2020, 410, pp.109342. 10.1016/j.jcp.2020.109342 . hal02860030

\section{HAL Id: hal-02860030 \\ https://hal.science/hal-02860030}

Submitted on 8 Jun 2020

HAL is a multi-disciplinary open access archive for the deposit and dissemination of scientific research documents, whether they are published or not. The documents may come from teaching and research institutions in France or abroad, or from public or private research centers.
L'archive ouverte pluridisciplinaire HAL, est destinée au dépôt et à la diffusion de documents scientifiques de niveau recherche, publiés ou non, émanant des établissements d'enseignement et de recherche français ou étrangers, des laboratoires publics ou privés. 


\title{
A new efficient momentum preserving Level-Set/VOF method for high density and momentum ratio incompressible two-phase flows
}

\author{
Davide Zuzio $^{\mathrm{a}, *}$, Annagrazia Orazzo ${ }^{\mathrm{a}}$, Jean-Luc Estivalèzes ${ }^{\mathrm{a}}$, Isabelle \\ Lagrange $^{\mathrm{a}}$ \\ ${ }^{a}$ Multi-Physics Department for Energetics, ONERA/DMPE - Université de Toulouse, \\ F-31055, Toulouse, France
}

\begin{abstract}
Many two-phase flows relevant to natural and industrial applications are characterized by high density ratios and high shears at the interface. These peculiarities make numerical simulations of such kind of flows still challenging. In particular, incompressible Level-Set/Volume-of-Fluid -based solvers are affected by the onset of diverging spurious velocities close to the interface. In this paper a new strategy, able to overcome these numerical instabilities, is described for staggered Cartesian grids. In order to achieve a consistent mass-momentum advection, a new auxiliary continuity equation is introduced and resolved along the momentum equations in the velocity control volumes. The mass fluxes are evaluated through the Volume-of-Fluid color function and directly used to calculate the momentum convective term. Several high-density test cases (the density ratio going from $10^{3}$ to $10^{6}$ ) are presented: the new algorithm shows significant improvements in stability and accuracy over the standard velocity based advection methods, together with a very low increase in computational time, estimated at $5 \% \div 10 \%$. Therefore, it is suitable to simulate more complex and realistic high density ratio two-phase flows, such as the breakup of a liquid jet in cross-flow here described.
\end{abstract}

\footnotetext{
* Davide Zuzio

Email addresses: Davide.Zuzio@onera.fr (Davide Zuzio), o.annagrazia@libero.it (Annagrazia Orazzo), Jean-Luc.Estivalezes@onera.fr (Jean-Luc Estivalèzes), Isabelle. Lagrange@onera.fr (Isabelle Lagrange)
}

Preprint submitted to Journal of Computational Physics

February 12, 2020 
Keywords: Two-phase flows, High density ratio, Coupled Level-Set Volume of Fluid, Navier-Stokes, Momentum conservation, Mass-Momentum consistency

\section{Introduction}

Two-phase flows are ubiquitous in industrial and scientific applications and, consequentially, they are the focus of many computational studies. Simulating such kind of flows is still a great challenge in particular because of the sharp jumps in fluid properties and the singular surface tension force that leads to a discontinuity in pressure across the interface. These discontinuities make particularly difficult the discretization of the governing equations.

Usually, the governing equations for single-phase incompressible flows are solved by the so-called projection method of Chorin [1]. In this method a temporary velocity field is first predicted from momentum equation by ignoring the pressure gradient. A pressure Poisson equation is solved to enforce a divergencefree velocity field by correcting the predicted velocity field. In order to facilitate the decoupling between velocity and pressure, a staggered grid (MAC, Marker And Cell) is generally used. This kind of grid makes trivial the discretization of the equations using finite difference or finite volume schemes ${ }^{1}$.

This method can be adapted to the two-phase flows in the one-fluid formulation (i.e. the various fluids are treated as one fluid with variables material properties, by taking into account proper jump conditions at fluids interface, see Tryggvason et al. [2]). Thus, a classical incompressible two-phase interfacial solver is built from the following steps:

- advection of the interface;

- evaluation of the fluid properties jumps at the interface;

- resolution of the prediction velocity equation;

\footnotetext{
${ }^{1}$ Finite volume schemes are however trickier to implement, as different overlapping control volumes are defined on a MAC grid
} 
- resolution of the Poisson pressure equation;

- update of the velocity field.

It is known that this kind of solvers suffers of numerical stability problems in case of high density and shear ratios between phases. Indeed, huge spurious velocities may appear near the interface leading to non physical results or, worse, to a diverging computation.

One reason may come from the resolution of the prediction equation in terms of velocity rather than in terms of momentum. As the velocity is continuous across the interface, this approach avoids the discretization of interfacial density discontinuity. As no momentum conservation is imposed, special techniques must be used to preserve the stability of the computation: accurate near-interface velocities must be defined by extension technique (Nguyen et al. [3]) in order to avoid errors in the momentum transfer (Sussman et al. [4], Xiao [5]). Raessi and Pitsch [6] were among the first to highlight the importance of a conservative formulation of momentum. They recalled the conclusion of Park et al. [7], who explicitly demonstrated the advantages of resolving a momentum prediction equation instead of a velocity equation.

The second reason comes from the staggered location of the grid. Pressure and phase indicator functions are located on the cell centers, while velocity components lie on the cell faces, see Fig. 1, leading to staggered control volumes for continuity and momentum equations: Rudman [8] highlighted the numerical errors arising from this particular situation, labelling as "inconsistent" the mass and momentum equations solved each one on its own control volume. Indeed, no relation exists between mass and momentum fluxes as they are not defined on the same faces.

The concept of numerical "consistency" in this particular problem has been referred to by several other authors. Raessi and Pitsch [6] defined: "Next, we further explain how using a consistent flux density yields a tight coupling between the mass and momentum transports."; Ghods and Herrmann [9] wrote: "Consistent mass and momentum advection was achieved by solving the conser- 
vative form of the momentum advection term with mass flux densities obtained from the volume-of-fluid-method."; and, finally, Owkes and Desjardins [10] specified: "All convective fluxes are computed with the same mass fluxes despite the staggered grid arrangement, which is required for a consistent and conservatives scheme". Within this work, "consistency" will therefore refer to the solving of continuity and momentum equations on the same control volumes, using the mass fluxes to compute the relative momentum fluxes.

A clever solution to the consistency problem has been proposed by Rudman [8]. The salient feature of Rudman's method is to get a consistent estimate of the density fluxes, namely $\rho \mathbf{V}$ (being $\rho$ the density and $\mathbf{V}$ the velocity vector), on the faces of each staggered control volume, which appear in the discretization of the convective terms $\nabla \cdot \rho \mathbf{V} \otimes \boldsymbol{V}$. To achieve consistency, a mesh twice as fine as the velocity-pressure grid is defined for the color function. This arrangement allows density fluxes to be known at the correct location, i.e. at a velocity cell face lying across two pressure cells faces. The mass fluxes are then used for both continuity and momentum equation, thus ensuring consistency.

This technique is nonetheless quite expensive, since a finer sub-grid is required. To avoid the sub-grid interface advection, Bussmann et al. [11] adapted the Rudman's method to unstructured collocated grid. An adaptation of the Rudman's algorithm to one grid for MAC variables collocation is presented in Vaudor et al. [12]. The main idea is to solve the mass balance on each half computational cell in order to evaluate the mass flux on the cell faces, where the velocity components are located, and then use the final PLIC-computed cellcentered density to estimate the unknown fluxes. Similarly, Fuster et al. [19] developed a CIAM-based VOF method in which the density fluxes are employed to compute relative momentum fluxes. To achieve consistency, the momentum equations are solved on each staggered control volume by recreating in each of those the necessary color fraction data.

LeChenadec and Pitsch [13] proposed another VOF approach to solve the conservative form of the incompressible two-phase NSE. Their method is based on a geometric VOF type integration of the convective flux of momentum that 


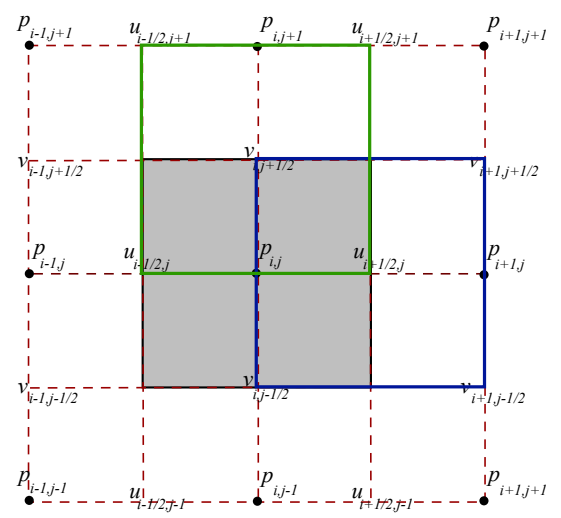

Figure 1: Schematic representation of a 2D staggered grid: pressure is cell-centered collocated while the velocity components are face-centered collocated. The grey area represents the pressure $p_{i, j}$ control volume, while the blue and the green squares define, respectively, the control volume considered for the $x$ and the $y$ velocity components, i.e. $u_{i+\frac{1}{2}, j}$ and $v_{i, j+\frac{1}{2}}$. Dashed lines delineate the sub-grid used by Rudman for VOF advection.

preserve monotonicity for the density, momentum and velocity fields. This treatment uses a Lagrange-Remap method, whose critical aspect is the deformation of the mesh cells during the remapping step to transfer the information from the Lagrangian to Eulerian coordinate system. To get around this problem, they triangulate each mesh elements, thus increasing a lot the computational cost. Owkes and Desjardins [10], instead, described an innovative, second order accurate, un-split and conservative VOF scheme, designed to guarantee consistency between the interface and the momentum transport through the construction of second-order density fluxes. Both the VOF and the momentum fluxes are geometrically treated by means of the construction of flux polyhedra. Nonetheless, the consistency is still achieved by a Rudman-like sub-grid approach, in which expensive un-split fluxes are computed on a twice finer mesh.

The problem of the inconsistencies between mass and momentum transport affects both VOF and Level-Set (LS) methods, see Scardovelli and Zaleski [14] and Sethian [15]. Many strategies were adapted to LS methods too. Raessi 
and Pitsch [6] used geometric arguments to compute the momentum-density fluxes by considering the LS field at two subsequent time instants in order to take into account the temporal evolution of the interface. This method is only limited to one- and two-dimension problems without a clear description of the construction of the non-diagonal fluxes. Desjardins and Moureau [16] developed two methodologies for improving high density ratio two-phase flows simulations. The first method is essentially based on an adaptation of Rudman's method to LS: they evaluate the density fluxes on the cell faces from the LS function advection scheme and, then, use these fluxes to construct the momentum fluxes. The second method, instead, is based on a two-velocity Ghost Fluid Method (GFM, for details on the original method see Fedkiw et al. [17]). Ghods and Herrmann [9] overcomes the generation of artificial momentum in presence of large density ratio in LS simulations by proposing a three-dimension algorithm applicable to the unstructured, collocated, finite volume formulation of NSE. They use a temporary mass balance discretized by operators consistent with those of the momentum equation, in order to obtain a predicted density ant thus recover the updated velocity. Their approach is neither mass nor momentum conservative: the key idea is to make mass and momentum solutions consistent.

Patel and Natarajan [18] proposed an hybrid staggered/non-staggered framework to solve multiphase flows on unstructured grids. They payed attention to the consistency of their continuity-momentum resolution by "invoking identical schemes for convective transport of volume fraction and momentum in the respective discrete equations". Their scheme has proven to be second-order accurate, bounded and stable for arbitrary high density ratios. The key aspect of their discretization is the resolution of a face-normal momentum equation on each cell face. The relative control volume is composed by the union of the two cells sharing the face, so that, on all the faces, continuity fluxes are known from the cell-centered continuity equation.

In this paper a numerical method to efficiently and consistently solve the incompressible, two-phase continuity and momentum equations on staggered (MAC) grids is proposed. The approach is based on a Coupled Level-Set/Volume- 
Of-Fluid Method (CLSVOF, Sussman and Puckett [20] and Menard et al. [21]), and it is meant to deal with high density ratio simulations with strong shearing flows, such as assisted atomization. The main idea is inspired by the work of Rudman [8], Ghods and Herrmann [9] and Desjardins and Moureau [16]. The salient feature of the method is to solve on each staggered momentum component control volume an auxiliary continuity equation to get a predicted density discretely consistent with the solution of momentum equation. To solve such continuity equation, the equivalence between mass and VOF highlighted by Rudman [8] is used. However, unlike the Rudman's method, the proposed approach does not require an additional twice finer grid, achieving similar consistency properties in a more efficient way: practical test will show an increase in CPU time of less than 10\% compared to a classical CLSVOF-velocity based solver. As the algorithm is based on a CLSVOF-PLIC approach, mass conservation is better than pure Level-Set methods. Relative mass errors are typically between $10^{-4} \div 10^{-14}$, a satisfying value for all practical purposes, although machine accuracy is seldom achieved.

The whole algorithm has been implemented and tested in the ONERA DYJEAT code (see Couderc [22], Zuzio and Estivalèzes [23], Zuzio et al. [24] and Xavier et al. [25]). Several test cases are presented, from simpler academical case to a realistic atomizations application. The results show the robustness of the solver for extremely high density ratios, as well as the ability to preserve liquid and gaz kinetic energies.

The paper is organized as follows: in $§ 2$ a review of the governing equations and of the numerical schemes is presented; in $§ 3$ the new algorithm is described in details; its capabilities are shown in $\S 4$ by means of some classical high density ratio test cases; then, an application to the atomization process is shown in a simulation of a water jet in cross-flow. $\S 5$ briefly illustrates some CPU performances of the new algorithm. Finally, some concluding remarks are drawn in $\S 6$. 


\section{Two-phases Navier-Stokes solver}

\subsection{Physical model}

The one-fluid formulation of the incompressible NSE, Eqs. (1)-(2), is here adopted. A single set of equations is used to describe the motion of the two fluids present in the domain, by locally defining the fluid properties:

$$
\begin{aligned}
\nabla \cdot \mathbf{V} & =0 \\
\frac{\partial \mathbf{V}}{\partial t}+\nabla \cdot(\mathbf{V} \otimes \mathbf{V}) & =\frac{1}{\rho}(\nabla \cdot \overline{\overline{\mathbf{T}}}+\mathbf{f})
\end{aligned}
$$

In this paper the vectors will be noted by bold symbols, the tensors with an additional double bar hat. $\mathbf{V}=[u, v, w]^{T}$ is the velocity field, f contains the

external forces like gravity, $\rho \mathbf{g}$. $\overline{\overline{\mathbf{T}}}$ is the stress tensor $\overline{\overline{\mathbf{T}}}=-p \overline{\overline{\mathbf{I}}}+\overline{\overline{\mathbf{D}}}$ where $p$ is the hydrodynamic pressure and $\overline{\overline{\mathbf{D}}}=\mu\left(\nabla \mathbf{V}+(\nabla \mathbf{V})^{T}\right)$ is the viscous stress. In the one-fluid formulation, both the density, $\rho(\phi, C)$, and the viscosity, $\mu(\phi, C)$, are function of the fluid occupying the computation cell, so they are function of the Level-Set, $\phi$, and of the VOF color function, $C$. The interface $\Gamma$ separates the two considered fluids, in our cases a gas, indicated by the subscripts $g$, and a liquid, indicated by the subscript $l$. In absence of phase change, the velocity field is continuous across $\Gamma$, i.e. $[\mathbf{V}]_{\Gamma}=0$, where $[\cdot]_{\Gamma}=(\cdot)_{l}-(\cdot)_{g}$ is the jump operator. The presence of the surface tension leads to a discontinuity in the normal stresses that is translated into a pressure jump, while the different viscosities impose a jump on the gradient of velocity:

$$
\begin{aligned}
\boldsymbol{n} \cdot\left[p I+\mu\left(\nabla \mathbf{V}+(\nabla \mathbf{V})^{T}\right)\right] \cdot \boldsymbol{n} & =\sigma \kappa \\
\boldsymbol{t} \cdot\left[\mu\left(\nabla \mathbf{V}+(\nabla \mathbf{V})^{T}\right)\right] \cdot \boldsymbol{n} & =0
\end{aligned}
$$

where $\sigma$ is the surface tension, $\kappa$ is the interface curvature, $\boldsymbol{n}$ is the interface normal vector and $\boldsymbol{t}$ the tangential one.

\subsection{Interface tracking}

In addition to the Navier-Stokes Eqs. (1)-(2), the kinematics of the gasliquid interface has to be solved as well, in order to identify at each time instant 
the fluids into the domain and to define their material properties. In this work the CLSVOF method described by Sussman and Puckett [20] and Menard et al. [21] is used ${ }^{2}$. Two advection equations have to be solved: one for the transport of the VOF color function, $C$, Eq. (5), and one for the LS function, $\phi$, Eq. (6).

$$
\begin{aligned}
& \frac{\partial C}{\partial t}+\mathbf{V} \cdot \nabla C=0 \\
& \frac{\partial \phi}{\partial t}+\mathbf{V} \cdot \nabla \phi=0
\end{aligned}
$$

The transport equation of $C$ is equivalent to the equation of mass conservation for an incompressible flow. Indeed, being $0 \leq C \leq 1$ a marker function defining the liquid volume present in each computational cell (if $C=1$ the cell is completely occupied by liquid while if $C=0$ the cell is completely occupied by gas), Eq. (5) represents under the condition (1) the conservation of the mass of each phase. The main idea of CLSVOF method is to benefit from the advantage of both LS and VOF strategies: the VOF method minimizes the mass loss, while the LS method keeps an accurate and smooth description of the geometrical properties of the interface, i.e. the interface normal vector, $\boldsymbol{n}$, and curvature, $\kappa$.

$$
\begin{aligned}
\boldsymbol{n} & =\frac{\nabla \phi}{\|\nabla \phi\|} \\
\kappa & =\nabla \cdot\left(\frac{\nabla \phi}{\|\nabla \phi\|}\right)
\end{aligned}
$$

Eq. (7) and (8) are discretized by second-order central differencing. The coupling between LS and VOF is based on the definition of the VOF color function, $C$, as a function of $\phi$, in each computational cell. Following the methodology presented in Sussman and Puckett [20] and Menard et al. [21], a PLIC (Piecewise Linear Interface Calculation) reconstruction of the interface $\phi^{R}$ in each mixed cell is performed from the existing Level-Set field $\phi$. The initial value of $C$ can be analytically computed by geometric relations, given here by means of the VoFTools of Lopéz and Hernandez [26], and then updated by Eq. (5).

\footnotetext{
${ }^{2}$ A WENO5-RK3 pure Level-Set approach from Couderc [22] is available as well, and is used for comparisons in $\S 4.1$ and $\S 5$
} 
The VoFTools package of FORTRAN subroutines provides geometrical and analytical tools to perform the two basic operations involved in a VOF method: the volume truncation and the enforcement of local volume conservation. The authors demonstrated that these tools reduce the difficulties associated with the highly complex geometrical operations involved in PLIC-VOF methods especially in $3 \mathrm{D}$.

The LS function and the VOF color function are advected simultaneously using a second-order Strang-Splitting from Strang [27]. The Eqs. (5)-(6) are solved in the following form proposed by Puckett et al. [28]:

$$
\begin{aligned}
& \frac{\partial C}{\partial t}+\nabla \cdot(\mathbf{V} C)=(\nabla \cdot \mathbf{V}) C, \\
& \frac{\partial \phi}{\partial t}+\nabla \cdot(\mathbf{V} \phi)=(\nabla \cdot \mathbf{V}) \phi,
\end{aligned}
$$

where $C$ and $\phi$ on the right-hand side are implicitly treated in each sweep step in order to ensure the discrete conservation of such quantities. Since $\phi$ is a smooth function, the fluxes presented in Eq. (10) are easily evaluated by means of a classic finite volume scheme (Sussman and Puckett [20]), while the $C$ fluxes are computed through geometrical computations, i.e. the polyhedron truncation procedure presented in Lopéz and Hernandez [26]. In this way the VOF fluxes represent exactly the amount of liquid crossing the cell faces. An example is reported in Fig. 2 where the evaluation of the 2D VOF flux on the right face of cell $(i, j), G_{i+\frac{1}{2}, j}^{x}$, is shown. If VOL is the volume of the liquid corresponding to the shaded trapezoid bordering the cell face at $i+\frac{1}{2}$, the flux crossing the cell face $\left(i+\frac{1}{2}, j\right)$ in the time unit $\Delta t$ and advected by the velocity $u_{i+\frac{1}{2}}$ is given by

$$
G_{i+\frac{1}{2}, j}^{x}=\frac{\mathrm{VOL}}{\left|u_{i+\frac{1}{2}}\right| \Delta t \Delta y} \cdot u_{i+\frac{1}{2}}
$$




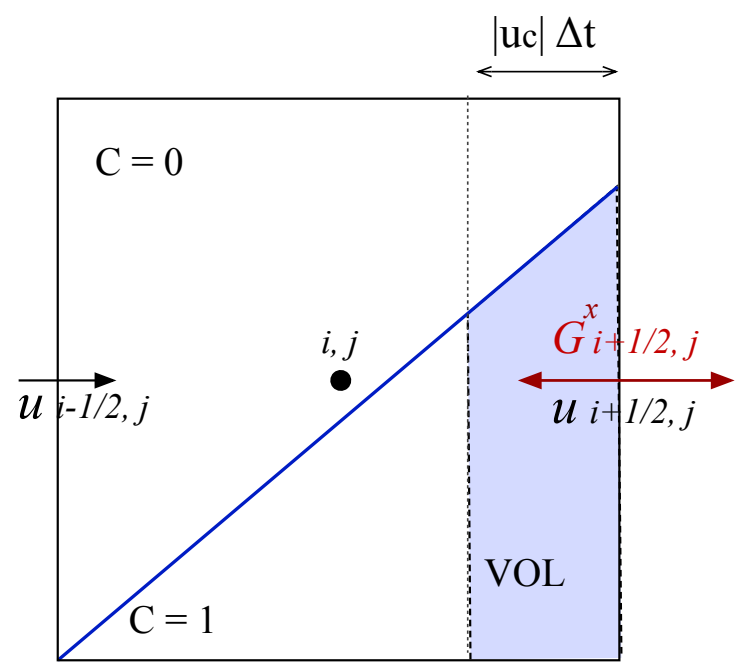

Figure 2: Geometric representation of the VOF flux in $x$-direction through the cell face $\left(i+\frac{1}{2}, j\right)$ in the case where $u_{i+\frac{1}{2}, j}>0$.

The updated VOF is used to offset the value of the LS in order to assure the local and global mass conservation.

At the end of each interface advection step $t^{n}+\Delta t \rightarrow t^{n+1}$, the $C^{n+1}$ and $\phi^{n+1}$ field are obtained. The updated fluids material properties $\rho^{n+1}$ and $\mu^{n+1}$ can then be evaluated, and used to solve Eq. (2) as well as compute the jump conditions. Details on the actual computation of mixed cells density will be given in $\S 3$ : the PLIC reconstruction $\phi^{R, n+1}$ allows the accurate computation of density on any control volume by using simple arithmetic averaging:

$$
\rho=C \rho_{l}+(1-C) \rho_{g}
$$

The pressure-velocity de-coupling is detailed in the following section.

\subsection{Navier-Stokes solver}

As stated in $\S 1$, the one-fluid formulation of NSE allows to use the numerical methods developed for single-phase flows with the interface tracking of 
CLSVOF. In the DYJEAT code, the governing equations are solved by means of the classical Chorin's projection method (see Chorin [1] for details) on staggered Cartesian uniform meshes. Within a time-step (or a Runge-Kutta subiteration), the interface tracking and the projection steps are performed sequentially: Eq.s (10) and (9) are solved to obtain the interface location and thus the material properties at $t^{n+1}$. Subsequently, the NSE solve is performed.

The first step of the Chorin's projection method provides a non divergencefree velocity field by resolving the so-called prediction Eq. (13), obtained from momentum Eq. (2) without considering the pressure term:

$$
\mathbf{V}^{*}=\mathbf{V}^{n}+\Delta t\left(\mathbf{V}^{n} \cdot \nabla \mathbf{V}^{n}+\frac{1}{\rho^{n+1}} \nabla \cdot\left(2 \mu^{n+1} \overline{\overline{\mathbf{D}}}^{n}\right)+\frac{1}{\rho^{n+1}} \mathbf{f}\right)
$$

The original non-consistent discretization of Eq. (13) is a finite-difference discretization using fifth-order WENO schemes for the convective terms.

The viscous term is treated explicitly and is here discretized by means of the method presented by Kang et al. [29]. The effects of the surface tension are taken into account in the Poisson equation by means of the GFM Fedkiw et al. [17] through the discretization of the pressure jump across the interface. In the GFM, ghost cells are defined on each side of the interface and appropriate schemes are applied to extrapolate the jump conditions. In this way the interface is treated as a sharp discontinuity and more accurate schemes are used for the discontinuous variable discretization. As the treatment of the viscous term described in Sussman et al. [4] is used, the pressure jump defined by the Eq. (3) turns into $[p]_{\Gamma}=\sigma \kappa$, the jump of the normal component of the viscous stress being already taken into account in the prediction equation. The pressure is evaluated by solving the elliptic equation

$$
\nabla \cdot\left(\frac{1}{\rho^{n+1}} \nabla p^{n+1}\right)=\frac{\nabla \cdot \mathbf{V}^{*}}{\Delta t}
$$

This equation is solved by means of a Multigrid preconditioned Conjugate Gradient method (MGCG), from Tatebe [30]. Finally, the divergence-free velocity field is obtained by correcting the predicted one :

$$
\mathbf{V}^{n+1}=\mathbf{V}^{*}+\Delta t \frac{1}{\rho^{n+1}} \nabla p^{n+1}
$$


The projection steps are repeated twice in a fully coupled second-order RungeKutta predictor-corrector scheme, each sub-step re-evaluating the convective velocity used to advect the CLSVOF and giving the final velocity (see Rudman [8] and Vaudor et al. [12]):

$$
\begin{aligned}
& \mathbf{V}^{n+\frac{1}{2}}=\mathbf{V}^{n}+d t \mathbf{F}^{n} \\
& \mathbf{V}^{n+1}=\frac{1}{2} \mathbf{V}^{n+\frac{1}{2}}+\frac{1}{2} d t \mathbf{F}^{n+\frac{1}{2}}
\end{aligned}
$$

being $\mathbf{F}$ the discrete form of Eq. (13). The interested reader is referred to Couderc [22] and Zuzio et al. [24] for more details on the actual implementation in DYJEAT.

The new algorithm presented in $\S 3$ will replace the non linear convective terms of Eq. (13) with a momentum equation consistent with an auxiliary continuity equation. The continuous form for momentum pure advection can therefore be written as:

$$
(\rho \mathbf{V})^{*}=(\rho \mathbf{V})^{n}+\Delta t \nabla \cdot\left((\rho \mathbf{V})^{n} \otimes \mathbf{V}^{n}\right)
$$

\section{Consistent Mass-Momentum transport (CMOM)}

The objective of the consistent momentum transport (CMOM in this paper) is to provide a more robust estimation of the convective component of $V^{*}$ in Eq. (13) when dealing with high density ratio two-phases flows. As seen in the review of the existing methods $\S 1$, the convective term of momentum equation has at first to be written in the conservative form. In consequence, the result of this equation is an updated $(\rho V)^{*}$, one component in each MAC staggered location. Dividing this value by an opportune density $\rho^{*}$ should give the expected convective part of $V^{*}$.

One possible estimation for the velocity-cell-centered density $\rho^{*}$ could come from the reconstructed PLIC interface $\phi^{R, n+1}$ obtained after integration of Eq. (9). If the PLIC line (plane in three dimensions) is integrated in half-cells,

an integral value $\rho_{i \pm \frac{1}{2}}^{n+1}$ can be directly obtained by sum of the opportune contributions. However, using this value still leads to non-consistent momentum 
updates: a simple high-density ratio test cases like the one presented in section 4.1 shows a diverging kinetic energy plot and the development of non-physical velocities.

An effective solution of this problem of consistency (and independent of the concept of conservation) is presented by Ghods and Herrmann [9], where a temporary density $\rho_{c v}^{*}$, computed on the MAC staggered location, is used to compute each component of the predicted velocity, and then discarded. In the present approach, the temporary density equations are solved together with momentum equations in a CLSVOF-PLIC framework, assuring better conservation properties than a pure Level-Set approach. There is therefore no need for the twice finer grid proposed by Rudman [8], thus improving the efficiency of the algorithm with similar benefits.

The proposed momentum solving algorithm is based on the following steps:

- Definition of staggered temporary continuity equations.

- Definition of consistent staggered cells integral values.

- Definition of consistent staggered cells momentum fluxes.

- Velocity update.

Note that hereinafter the description of the new algorithm is given only for $2 \mathrm{D}$ case, the 3D extension being straightforward.

\subsection{Local mass equation}

The aforementioned idea is to solve an auxiliary continuity equation in each velocity component volume, V2 for $u$ and V3 for $v$ as shown in Fig. 3. This gives a local predicted density $\rho_{i}^{*}, i=u, v$, consistent with the corresponding predicted momentum $(\rho u)^{*}$ and $(\rho v)^{*}$, see Eq.s (18)-(21);

$$
\begin{aligned}
\rho_{u}^{*} & =\rho_{u}^{n}-\Delta t\left(\nabla \cdot\left(\rho_{u} \mathbf{V}\right)\right)^{n} \\
\left(\rho_{u} u\right)^{*} & =\left(\rho_{u} u\right)^{n}-\Delta t\left(\nabla \cdot\left(\rho_{u} \mathbf{V} u\right)\right)^{n} \\
\rho_{v}^{*} & =\rho_{v}^{n}-\Delta t\left(\nabla \cdot\left(\rho_{v} \mathbf{V}\right)\right)^{n} \\
\left(\rho_{v} v\right)^{*} & =\left(\rho_{v} v\right)^{n}-\Delta t\left(\nabla \cdot\left(\rho_{v} \mathbf{V} v\right)\right)^{n}
\end{aligned}
$$


In order to solve in a finite volume framework the resulting equations, integral cell-centered values and face fluxes must be defined.

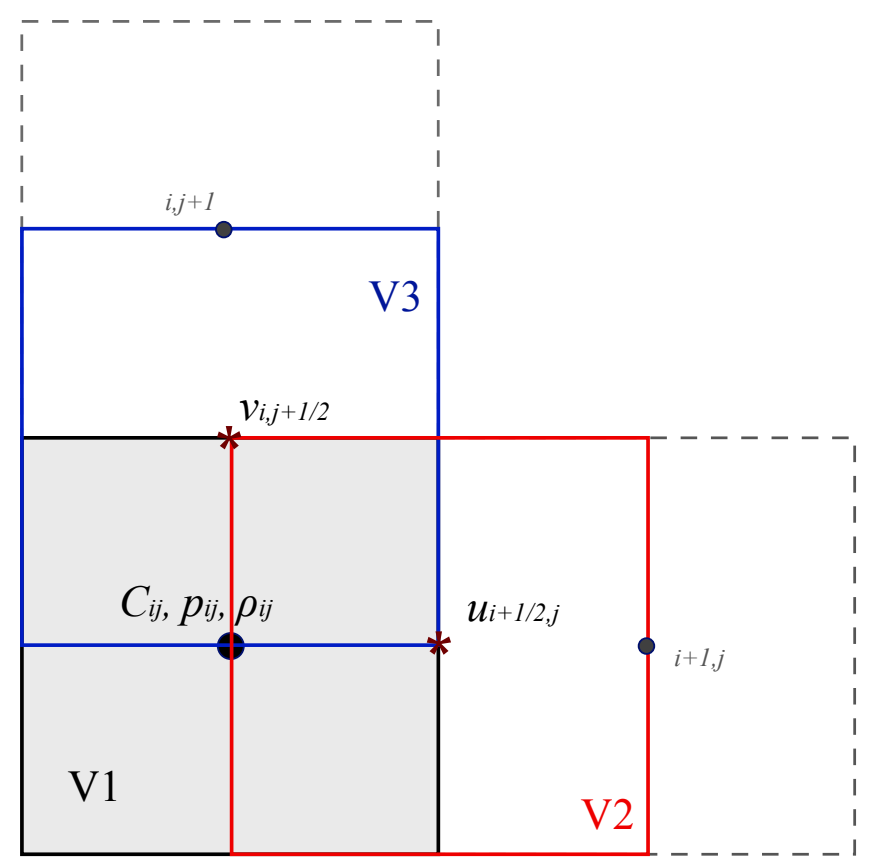

Figure 3: Representation of the different control volumes considered in a 2D staggered grid: V1 for the resolution of pressure/LS/VOF equation and V2 and V3, respectively, for $x$-component and $y$-component of momentum.

\subsection{Consistent cell integral values}

The solution of Eqs. (18)-(21) in momentum control volumes implies the determination of face-centered density values, $\rho_{u}$ and $\rho_{v}$ in Fig. 4 , defined at the same points where $u$ and $v$ are located. 


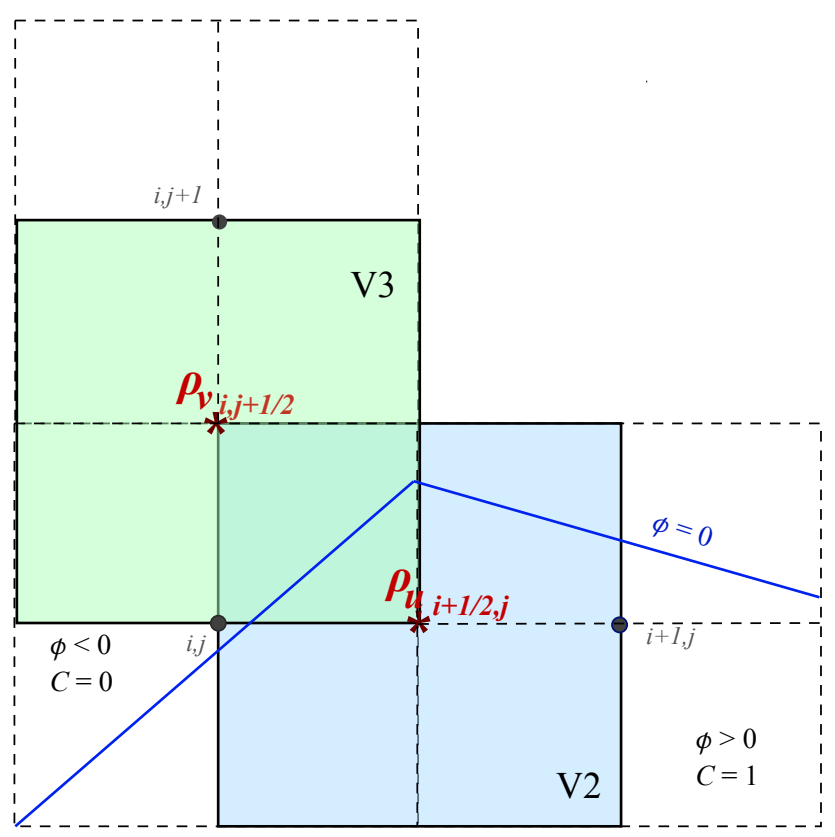

Figure 4: Definition of the face-centered density values to be used into momentum equations. The blue line represents the PLIC reconstruction $\phi^{R}$ on the pressure cell centered on $i, j$.

As a finite volume discretization of Eqs. (18)-(21) is desired, $\rho_{u}$ and $\rho_{v}$ have to be representative of the integral values in Eq.s (22)-(23):

$$
\begin{aligned}
\rho_{u} & =\frac{1}{\mathrm{~V} 2} \int_{\mathrm{V} 2} \rho(\boldsymbol{x}) d \Omega \\
\rho_{v} & =\frac{1}{\mathrm{~V} 3} \int_{\mathrm{V} 3} \rho(\boldsymbol{x}) d \Omega
\end{aligned}
$$

Following the idea of Rudman [8], the color function $C$ is computed on the quadrants of a quad(oct)-tree subdivision of each mixed cell (four different values of $C$ are derived in 2D and eight in 3D for each computational cell). These values are obtained from the PLIC reconstruction at the time step $n, \phi^{R, n}$, using the polyhedron truncation procedure provided by VoFTools (the same on which is based the resolution of Eq. (9)). A sub-grid density is therefore computed 
using the arithmetic average of Eq. (12) on each sub-volume. The sought V2-V3 integral densities are given by sum of the opportune contributions: an example is given by Eqs. (24)-(25), whose control volume is described in Fig. 5.

$$
\begin{aligned}
& \rho_{u_{i+\frac{1}{2}, j}}^{n}=\frac{1}{4}\left(\rho_{i+\frac{1}{4}, j+\frac{1}{4}}^{n}+\rho_{i+\frac{1}{4}, j-\frac{1}{4}}^{n}+\rho_{i+\frac{3}{4}, j+\frac{1}{4}}^{n}+\rho_{i+\frac{3}{4}, j-\frac{1}{4}}^{n}\right) \\
& \rho_{v_{i, j+\frac{1}{2}}}^{n}=\frac{1}{4}\left(\rho_{i-\frac{1}{4}, j+\frac{1}{4}}^{n}+\rho_{i+\frac{1}{4}, j+\frac{1}{4}}^{n}+\rho_{i-\frac{1}{4}, j+\frac{3}{4}}^{n}+\rho_{i+\frac{1}{4}, j+\frac{3}{4}}^{n}\right)
\end{aligned}
$$

Differently from the Rudman's algorithm, no subgrid reconstruction is needed to calculate the sub-cells densities appearing in Eq.s (24) and (25). Indeed, as can be seen in Fig. 5, the linear PLIC reconstructions already known in the main cells $i, j$ and $i+1, j$ are used to evaluate the volume fraction in the sub-cells, shown by the shaded area: $C_{i+\frac{1}{4}, j+\frac{1}{4}}^{n}, C_{i+\frac{1}{4}, j-\frac{1}{4}}^{n}, C_{i+\frac{3}{4}, j+\frac{1}{4}}^{n}, C_{i+\frac{3}{4}, j-\frac{1}{4}}^{n}$. 


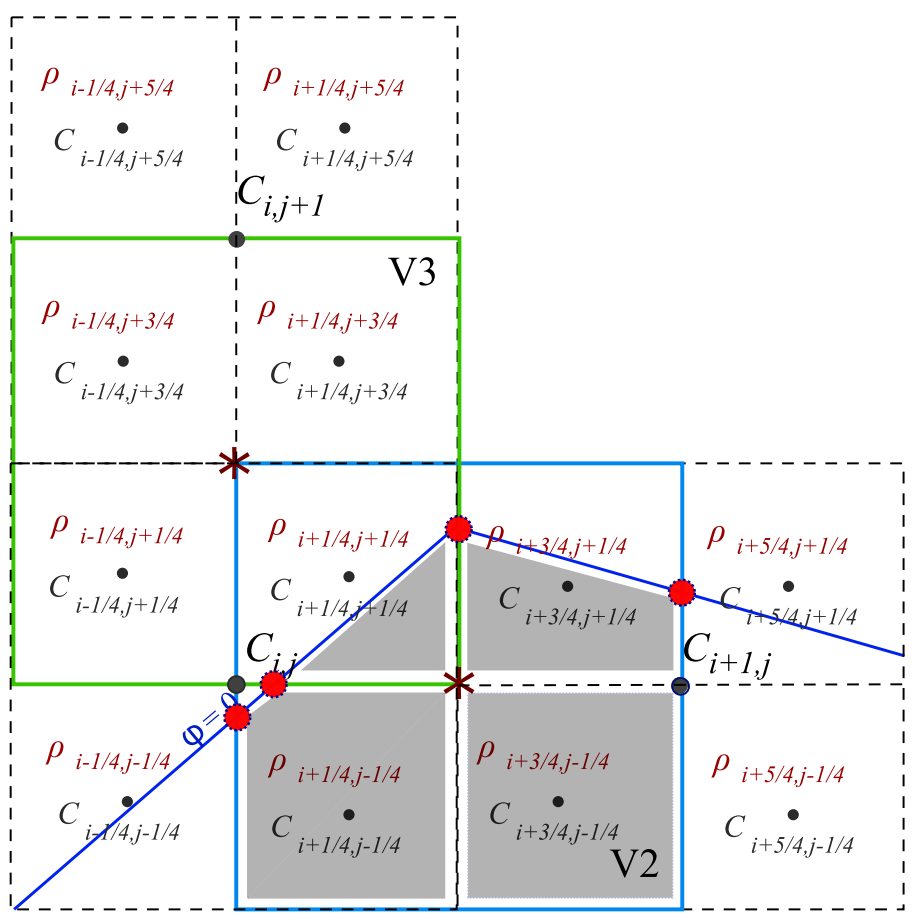

Figure 5: Sub-grid VOF reconstruction and density values definition. The blue segments represent the PLIC reconstructions $\phi^{R}$ on the pressure cells $i, j$ and $i+1, j$. The shaded areas correspond to the sub-grid volume fraction used to compute subgrid densities in Eq.s (24) and (25). The red marks show the intersections between the PLIC reconstructions and the sub-cell faces.

From these sub-cells volume fractions, the corresponding densities are computed using the generic Eq. (12). Therefore the main extra cost of the proposed approach is the computation of the PLIC reconstructed intersection (the red dots in Fig. 5) with the sub-cells, which was found nearly negligible. The face-centered integral densities are advected along the momentum components, which are themselves the product between the face-centered densities and the face-centered velocity components $(u, v)$, following Eq.s (18)- (21) in $x$ - and $y$-directions.

The following step is to evaluate the numerical fluxes on the staggered cells. 


\subsection{Consistent flux evaluation}

Assumed the equivalence between mass and VOF, the density fluxes, $G^{m}=$ $\rho \mathbf{V}$, can be directly derived from the VOF fluxes $G$. For example, at $i+\frac{1}{2}$ cell face, the flux is defined as:

$$
G_{i+\frac{1}{2}, j}^{m}=G_{i+\frac{1}{2}, j} \rho_{l}+\left(1-G_{i+\frac{1}{2}, j}\right) \rho_{g}
$$

where $u_{i+\frac{1}{2}, j}$ is the face-centered convective speed. To evaluate such VOF fluxes, the same geometrical computations used to resolve Eq. (9) in $§ 2.2$ are considered but, in this contest, they are applied to different control volumes (i.e. V2 or V3 instead of V1). Hence, for example, considering the equations in the $x$-direction, Eqs. (18)-(19), to compute the $x$-component of density flux $\rho_{u} \mathbf{V}$ through the face $(i, j)$ of V2, $G_{i, j}^{m, x}$ in Fig. 6 , the VOF flux, $G_{i, j}^{x}$, is required

$$
G_{i, j}^{x}=\frac{\mathrm{VOL}}{\left|u_{c}\right| \Delta t \Delta y} \cdot u_{c}
$$

where, if the convective speed $u_{c}=\frac{1}{2}\left(u_{i-\frac{1}{2}, j}+u_{i+\frac{1}{2}, j}\right)$ is positive, VOL is the liquid volume limited by the dotted line of Fig. 6 . In the same way is computed $G_{i+1, j}^{m, x}$, for which the convective velocity is $u_{c}=\frac{1}{2}\left(u_{i+\frac{1}{2}, j}+u_{i+\frac{3}{2}, j}\right)$

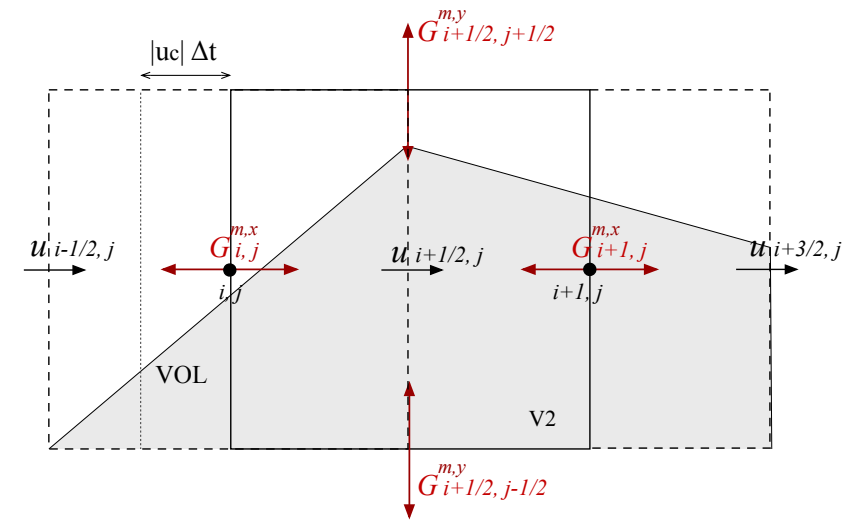

Figure 6: Representation of the mass fluxes in the $u$ control volume, i.e. V2. 
The evaluation of the $\rho_{u}$ fluxes in $y$-direction $\left(\rho_{u} v_{c}\right), G_{i+\frac{1}{2}, j \pm \frac{1}{2}}^{m, y}$ in Fig. 7, is a bit trickier. Each one of these can be evaluated as the sum of two fluxes crossing a face of dimension $\frac{\Delta x}{2}$

$$
G_{i+\frac{1}{2}, j \pm \frac{1}{2}}^{m, y}=\frac{G_{i+\frac{1}{4}, j \pm \frac{1}{2}}^{m, y}+G_{i+\frac{3}{4}, j \pm \frac{1}{2}}^{m, y}}{2}
$$

For example, the VOF fluxes necessary to estimate $G_{i+\frac{1}{4}, j-\frac{1}{2}}^{m, y}$ and $G_{i+\frac{3}{4}, j-\frac{1}{2}}^{m, y}$, are given by

$$
\begin{aligned}
G_{i+\frac{1}{4}, j-\frac{1}{2}}^{y} & =\frac{\mathrm{VOL}}{\left|v_{i, j-\frac{1}{2}}\right| \Delta t \frac{\Delta x}{2}} \cdot v_{i, j-\frac{1}{2}} \\
G_{i+\frac{3}{4}, j-\frac{1}{2}}^{y} & =\frac{\mathrm{VOL}}{\left|v_{i+1, j-\frac{1}{2}}\right| \Delta t \frac{\Delta x}{2}} \cdot v_{i+1, j-\frac{1}{2}}
\end{aligned}
$$

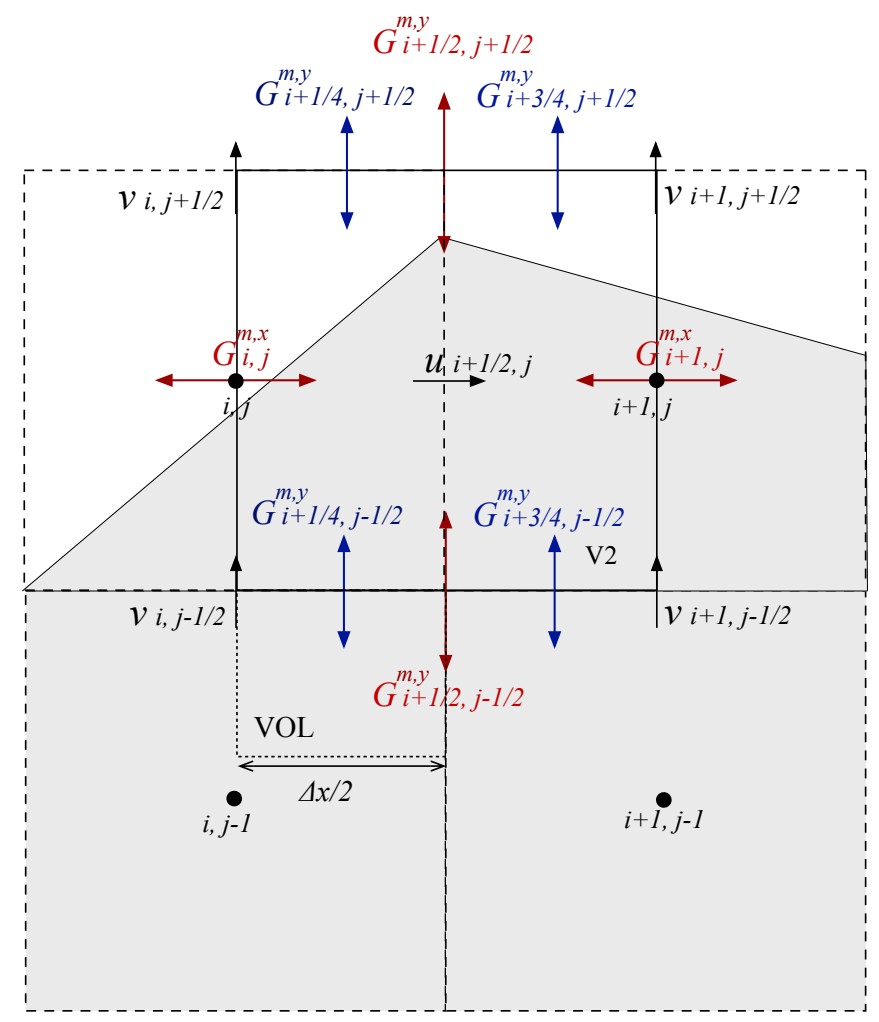

Figure 7: Evaluation of the $x$-component mass flux in $y$-direction, $\rho_{f c_{x}} v_{c}$. 
The momentum convective fluxes can be deduced at the same time of mass fluxes by simply multiplying the latter for the appropriate advected velocity component. Eq.s (31) and (32) show the update of both the $x$-component of momentum and the associated auxiliary continuity equation, both discretized on V2:

$$
\begin{array}{r}
\left(\rho_{u}\right)_{i+\frac{1}{2}, j}^{*}=\left(\rho_{u}\right)_{i+\frac{1}{2}, j}^{n}-\Delta t\left[\left(\frac{G_{i+1, j}^{m, x}-G_{i, j}^{m, x}}{\Delta x}\right)\right. \\
\left.+\left(\frac{G_{i+\frac{1}{2}, j+\frac{1}{2}}^{m, y}-G_{i+\frac{1}{2}, j-\frac{1}{2}}^{m, y}}{\Delta y}\right)\right]^{n+1} \\
\left(\rho_{u} u\right)_{i+\frac{1}{2}, j}^{*}=\left(\rho_{u} u\right)_{i+\frac{1}{2}, j}^{n}-\Delta t\left[\left(\frac{G_{i+1, j}^{m, x} \tilde{u}_{i+1, j}-G_{i, j}^{m, x} \tilde{u}_{i, j}}{\Delta x}\right)\right. \\
\left.+\left(\frac{G_{i+\frac{1}{2}, j+\frac{1}{2}}^{m, y} \tilde{u}_{i+\frac{1}{2}, j+\frac{1}{2}}-G_{i+\frac{1}{2}, j-\frac{1}{2}}^{m, y} \tilde{u}_{i+\frac{1}{2}, j-\frac{1}{2}}}{\Delta y}\right)\right]^{n+1}
\end{array}
$$

Actually, since at each time-step the LS and VOF fields are available both at $t^{n}$ and at $t^{n+1}$, it is possible to evaluate the fluxes at different time instants, i.e. $G_{u}^{n}, G_{u}^{n+1}$ and $G_{u}^{n+\frac{1}{2}}$. Practical test have shown that using the updated $\phi^{R, n+1} \rightarrow G_{u}^{n+1}$ reconstruction gives more accurate results than other choices. In Eq. (32), the advected $\tilde{u}$ speed is obtained by a high order WENO5.

\subsection{Velocity update}

Once the continuity and momentum equations are solved, the auxiliary predicted densities are used to recover the predicted velocity components as in Eq.s (33) and (34). The convective part of the predicted velocity, $V^{*}$, is finally computed as:

$$
\begin{aligned}
& u_{i+\frac{1}{2}, j}^{*}=\frac{\left(\rho_{u} u\right)_{i+\frac{1}{2}, j}^{*}}{\left(\rho_{u}\right)_{i+\frac{1}{2}, j}^{*}} \\
& v_{i, j+\frac{1}{2}}^{*}=\frac{\left(\rho_{v} u\right)_{i, j+\frac{1}{2}}^{*}}{\left(\rho_{v}\right)_{i, j+\frac{1}{2}}^{*}}
\end{aligned}
$$

The auxiliary densities $\rho^{*}$ are discarded at the end of each time-step. 
In order to compute $\mathbf{V}^{n+1}$, the viscous part and the external forces have still to be added to $\mathbf{V}^{*}$, see Eq. (13). Subsequently, the predicted velocity $\mathbf{V}^{*}$ is projected into the subspace of divergence-free velocity after solving the pressure Poisson equation, Eq.s (14) and (15). An important point is that the coefficient $\frac{1}{\rho^{n+1}}$ appearing in the pressure equation has to be computed by the face-centered densities evaluated by means of Eqs. (24)-(25), considering the VOF field at time $t^{n+1}$. Indeed, the source term of the pressure equation Eq. (14) depends on the face-centered densities, as it is obtained by computing the divergence of the predicted velocity field $\mathbf{V}^{*}$. In the CLSVOF-WENO formulation, these densities are obtained by Level-Set $\phi^{n+1}$ based interpolations. In the CMOM algorithm, any definition of the face-centered density values other than the one coming from Eqs. (24)-(25) has shown to re-introduce consistency errors in the projection step, Eq. (15). For the time integration of governing equation, a second-order accurate Euler Predictor-Corrector scheme, already used in Rudman [8] and Vaudor et al. [12], has been implemented. This method is compatible with the geometric nature of the mass/momentum convective fluxes and consists in two Euler steps. In the first step, the predictor step, the time integration is performed on half time step, $\Delta t / 2$, in order to find the velocity $\mathbf{V}^{n+\frac{1}{2}}$. Such velocity is then used in a second Euler step, the corrector step, in which the full time step is considered, to approximate the convective velocity as well as to compute the viscous stress tensor.

\section{Validations and Results}

\subsection{Transport of a very-high density fluid sphere}

This simple test case, originally proposed in Bussmann et al. [11], involves the motion of a high-density fluid sphere, $\rho_{\mathrm{h}}$, in a still lower-density fluid, $\rho_{\mathrm{l}}$. Purposefully, no surface tension nor viscous effects are taken into account: only the convective and pressure terms contribute to the momentum equation. The density ratio is set to be arbitrarily high, $10^{6}$ in most literature examples (Ghods and Herrmann [9], Raessi and Pitsch [6], LeChenadec and Pitsch [13], Vaudor 
et al. [12]). Given the high density ratio, the influence of the ambient fluid diminishes and the solution eventually approaches the rigid translation of the sphere in a void. The behaviour of the solver in this test is therefore heavily influenced by the discretization of the non linear convective terms of Eq. (17). In particular, any inconsistency between the mass and momentum transport is quickly amplified by the density jump, leading to erroneous deformations of the sphere.

As the numerical method proposed in this paper is based on a split VOF formulation, two different tests are presented. In the first, called the X-direction case, the sphere is set in motion along the $x$-axis, so that the only non-zero mass and momentum fluxes for the high-density fluid are those in the same direction, effectively removing any error coming from the directional splitting. In the second, called the XYZ-direction case, the initial velocity of the sphere is set with all non-zero components, thus imposing a diagonal motion. Any splitting effect should therefore appear in the second test case.

All the parameters in this test case are given in a non dimensional form. The sphere, of radius $R=0.15$ and density $\rho_{\mathrm{h}}=10^{6}$, is initially located at the center of a cubic periodic domain of side $L=1$. An initial velocity $\mathbf{V}_{0}^{X}=(10,0,0)$ or $\mathbf{V}_{0}^{X Y Z}=(10,10,10)$ is set inside the heavy fluid sphere, while the lowdensity phase of density $\rho_{1}=10^{0}$ is at rest. The theoretical solution of the simulation is the returning of the sphere to its initial position, after $t^{\text {end }}=0.1$. In consequence, the simulations are performed up to a full transit time, i.e. until the sphere has returned to its initial position. The results will be presented in non dimensional time $t / t^{\text {end }}$. The examined results are the global shape, the position error, and the evolution of the kinetic energy. As no gravity field is imposed, the totality of the energy contained in the domain corresponds to the initial kinetic energy. The reference values are the initial shape and position, while the kinetic energy should prove to be conserved as the computation is carried on. 
Comparison of numerical methods.

In this first set of simulations, performed in the $x$-direction, the classical velocitybased WENO approaches used in the previous releases of DYJEAT, namely the Level-Set/WENO (LS), an iso-contour correction variant (LS(ISO)) (as defined in Couderc [22]) and the Coupled Level-Set/Volume-of-Fluid/WENO (CLSVOF) are compared to the proposed Consistent Momentum approach (CMOM). A fixed mesh of $32^{3}$ is used, resulting in approximately 10 grid points per diameter. This low resolution has been intentionally chosen as poorly solved droplets appear routinely in atomization simulations. Mesh refinement effects are shown in the following section for the CMOM scheme.

Fig. 8 presents a visualization of the four simulations results, namely the Level-Set zero contour at $t^{\text {end }}$, or a full transit. The initial condition is drawn in grey; it represents for this test case the reference solution as well.

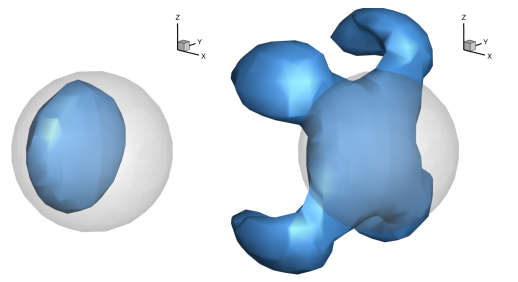

(a) LS

(b) LS-iso

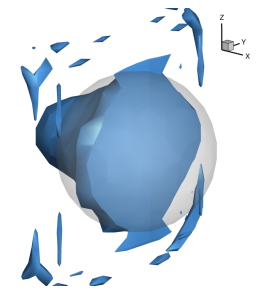

(c) CLSVOF

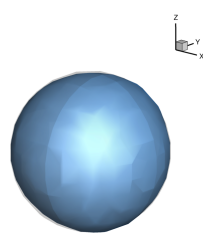

(d) CMOM

Figure 8: Sphere shapes after a full transit. Greyed out is the initial condition.

It is clear that all the previous velocity based formulations of the momentum equation fail this test. The base Level-Set/WENO method loses much of the initial mass, while the iso-contour correction erroneously adds mass in nonphysical deformations. The CLSVOF/WENO is only marginally more effective in preserving a spherical shape. The proposed scheme is conversely able to effectively maintain the initial shape. Fig. 9 presents the time evolution of the normalized high density phase mass and kinetic energy. These values are computed at each time-step. In the following computation, $C_{i, j, k}(t)$ is the VOF volume fraction in the $(i, j, k)^{t h}$ cell at the time $t$ and $C_{i, j, k}^{0}=C_{i, j, k}(t=0)$ the 
initial condition:

$$
\frac{M(t)}{M_{0}}=\frac{\sum_{i, j, k} C_{i, j, k}(t)}{\sum_{i, j, k} C_{i, j, k}^{0}} .
$$

The kinetic energy is trickier to compute, as within the MAC discretization it has to be computed by interpolating velocity components on the cell centers. A cell-centered normalized kinetic energy variable is thus reconstructed as follows:

$$
\begin{aligned}
\mathbf{V}_{i, j, k}^{x, c c} & =\left(u_{i, j, k}+u_{i-1, j, k}\right) / 2, \\
\mathbf{V}_{i, j, k}^{y, c c} & =\left(v_{i, j, k}+v_{i, j-1, k}\right) / 2, \\
\mathbf{V}_{i, j, k}^{z, c c} & =\left(w_{i, j, k}+w_{i, j, k-1}\right) / 2,
\end{aligned}
$$

and subsequently by integrating on the whole computational domain as

$$
K=\frac{E_{k}(t)}{E_{k}^{0}}=\frac{\sum_{m} \sum_{i, j, k}\left(\rho_{i, j, k}(t) \mathbf{V}_{i, j, k}^{m, c c}(t)\right)^{2}}{\sum_{m} \sum_{i, j, k}\left(\rho_{i, j, k}^{0} \mathbf{V}_{i, j, k}^{m, c c, 0}\right)^{2}}
$$




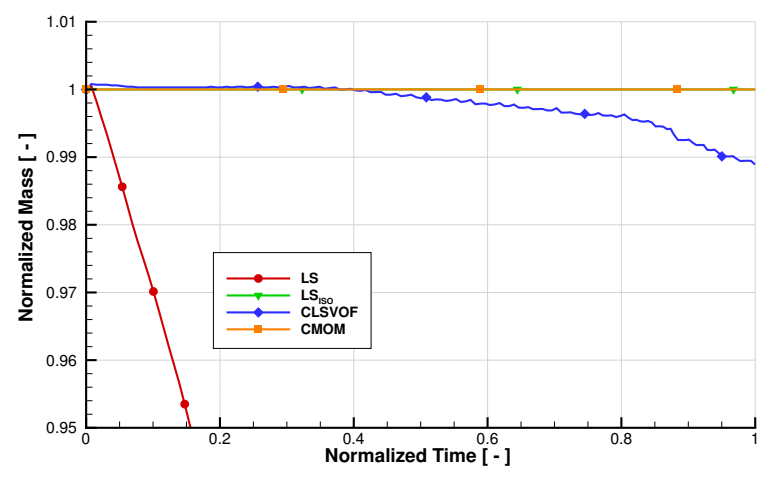

(a) Normalized mass

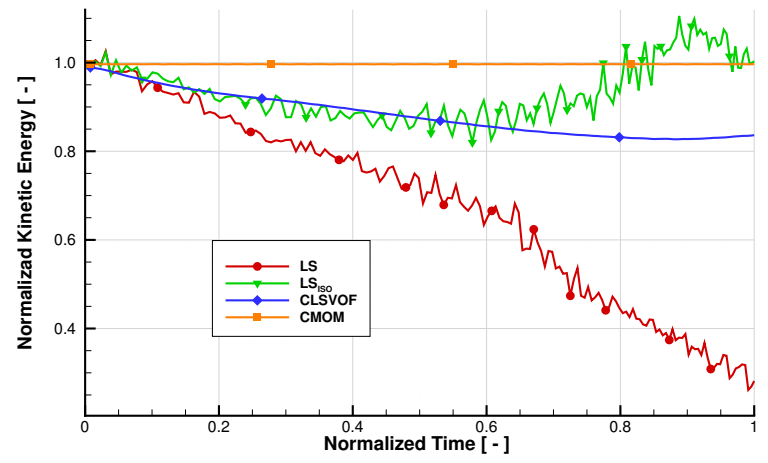

(b) Normalized kinetic energy

Figure 9: Time history of mass and energy evolution for the high density ratio sphere convection. Several numerical methods: (LS) Level-Set/WENO; (LS-iso) Level-Set Iso-Contour/WENO;(CLSVOF) Coupled Level-Set/VOF/WENO; (CMOM) Conservative momentum.

the density being evaluated as usually done by $\rho_{i, j, k}=\rho_{l} C_{i, j, k}+\rho_{g}\left(1-C_{i, j, k}\right)$. It should be pointed out, however, that this energy is a derived variable involving arbitrary interpolations, so an exact conservation of the integral value is not expected. A nearly constant value will be taken as a successful result from the computations, as what the method aims to avoid are artificial energy sources or dissipation due to incorrect $\rho \mathbf{V}$ products. Fig. 9a shows the evident Level-Set 
loss of mass, and while the iso-correction imposes a forced conservation, it has been observed the strong non-physical sphere deformation. The CLSVOF behaves better, but the loss of the spherical shape seen in Fig. 8 starts to induce a mass reduction towards the end of the sphere translation, of about $0.01 \%$. It is clear that further simulation time would lead all these methods to diverging results. The proposed CMOM, on the other side, allows mass conservation up to the eleventh decimal, in agreement with the visual qualitative analysis. The kinetic energy plot, Fig. 9b, shows a similar behaviour: the Level-Set/WENO methods show inconsistent energy values, the classical CLSVOF/WENO exhibits a steady dissipative behaviour linked to the mass loss, while the proposed CMOM finds out a steady kinetic energy value, with low level fluctuations of amplitude lower than $10^{-5} \times K_{0}$.

CMOM: splitting and mesh refinement effects.

In this paragraph a more in-depth analysis of the CMOM scheme is presented. In particular, mesh refinement and directional splitting effects are analysed. Two test cases, X-aligned and XYZ-diagonal transport, are repeated for four increasing meshes, $32^{3}, 64^{3}, 128^{3}$ and $256^{3}$. This corresponds to roughly 10,20 , 40 and 80 mesh cells per diameter. As already said in the introduction of this section, the very high density ratio allows to consider the motion of the sphere as a rigid translation. In a periodic domain, the sphere should maintain its initial velocity and therefore return to its initial position after a full flow-through. On the other hand, the gas velocity field is supposed to be heavily perturbed by the droplet movement. The computed liquid position and velocity at $t=t^{\text {end }}=0.1$ can be therefore compared to the initial conditions to evaluate the advection errors, induced by the numerical scheme. The initial velocity field is shown in Fig. 10a on the $z=0$ slice. The initial field involves $\mathbf{V}_{0}^{X}=(10,0,0)$ inside the liquid and a two-cells halo away from the interface to avoid any initial liquid momentum error, and zero elsewhere. The final field is shown in Fig. 10b. The velocity inside the droplet kept its initial value. The gas velocity adapts to the droplet movement to preserve continuity and momentum, thus generating a non- 
viscous wake. As the simulation is non-viscous, the fluid far from the droplet remains therefore almost undisturbed. Fig. 11 presents the mesh convergence effect on the velocity and density fields. For all the considered meshes, the initial $10 \mathrm{~m} . \mathrm{s}^{-1}$ field is correctly conserved, Fig. 11a. The density jump, Fig. 11b, is well captured by the Level-Set/VOF approach and shows the profile sharpening with the increasing resolution.

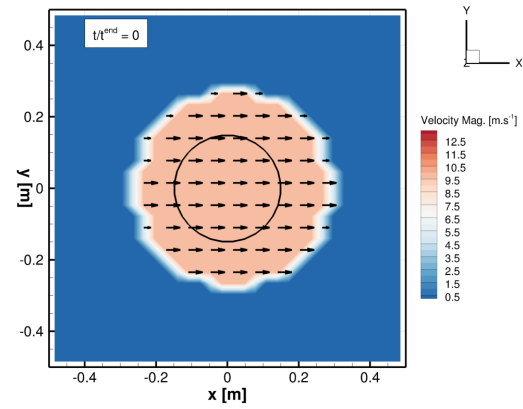

(a)

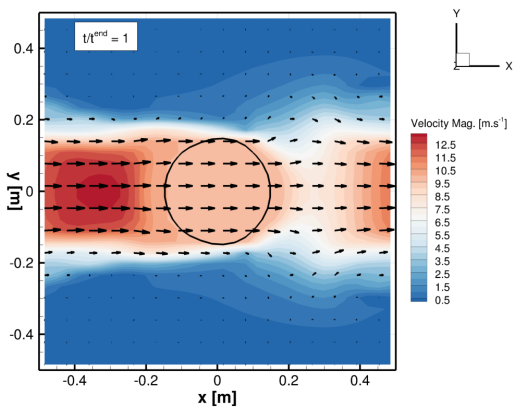

(b)

Figure 10: Velocity magnitude fields and vectors for the $32^{3}$ mesh on the $z=0$ slice, at (a) initial and (b) final times. The droplet shape is outlined in black. Only some of the vectors are shown for the sake of clarity.

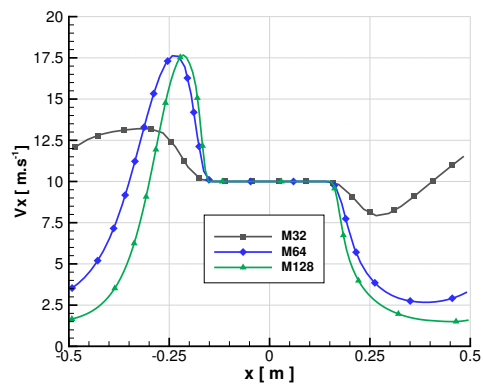

(a)

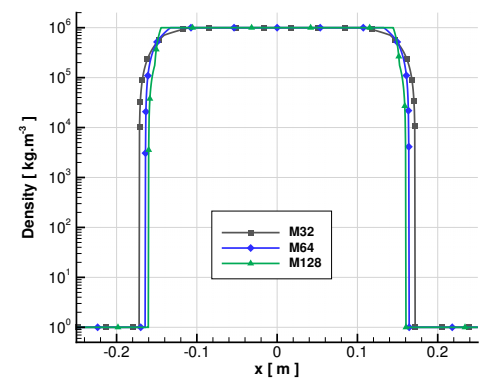

(b)

Figure 11: (a) x-velocity and (b) density profiles for three meshes, $32^{3}, 64^{3}$ and $128^{3}$, at the final simulation time $t^{\text {end }}$. 
In order to quantify the performances of the method, the following error norms are defined:

$$
\begin{aligned}
\|E r r\|_{1} & =\sum_{i, j, k}\left|\phi_{i, j, k}^{t^{\text {end }}}-\phi_{i, j, k}^{0}\right| \\
\|E r r\|_{2} & =\left[\sum_{i, j, k}\left(\phi_{i, j, k}^{t^{\text {end }}}-\phi_{i, j, k}^{0}\right)^{2}\right]^{\frac{1}{2}} \\
\|E r r\|_{\infty} & =\max \left|\phi_{i, j, k}^{t^{\text {end }}}-\phi_{i, j, k}^{0}\right|
\end{aligned}
$$

as well as the relative convergence rate:

$$
q=\frac{\ln \left(\operatorname{Err}_{2 \Delta x}\right)-\ln \left(\operatorname{Err}_{\Delta x}\right)}{\ln (2)}
$$

Tables 1 and 2 and Fig. 12 present the error norms and the relative convergence rates. Increasing the mesh improves the quality of the results, the convergence rates being bracketed between the first and second order. A closer look at the graph shows the better accuracy of the numerical method for the X-direction transport, a foreseen behaviour as no splitting approximation is performed: all the $\mathrm{Y}$ and $\mathrm{Z}$ fluxes are theoretically equal to zero for the dense phase. However, the gap seems to shrink for higher resolutions, as shown by the higher convergence rates of the XYZ-direction transport. 


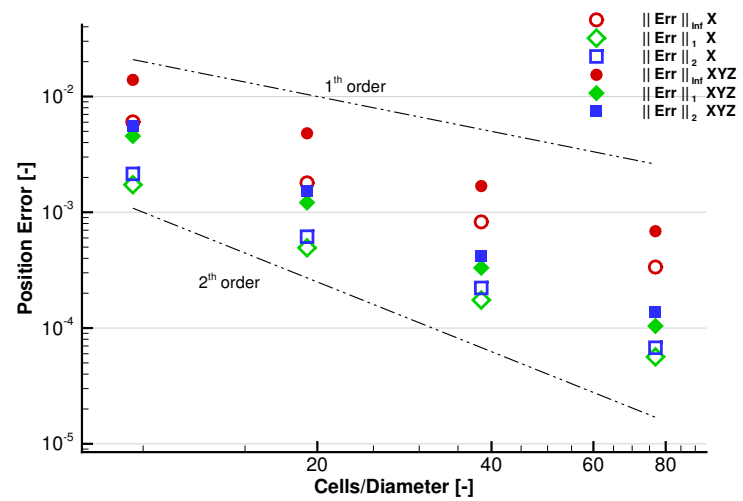

Figure 12: Position error of the sphere after a full flow-through. Two test cases, Xdirection and XYZ-direction advections. Three error norms are compared to first and second-order mesh convergences.

\begin{tabular}{ccccccc}
\hline \multicolumn{7}{c}{ X-Transport position error norms } \\
\hline Grid & $\|$ Err $\|_{1}$ & $q_{1}$ & $\|\operatorname{Err}\|_{2}$ & $q_{2}$ & $\|\operatorname{Err}\|_{\infty}$ & $q_{\infty}$ \\
\hline 32 & $1.73 \mathrm{e}-03$ & - & $2.15 \mathrm{e}-03$ & - & $6.04 \mathrm{e}-03$ & - \\
64 & $4.94 \mathrm{e}-04$ & 1.81 & $6.15 \mathrm{e}-04$ & 1.80 & $1.79 \mathrm{e}-03$ & 1.75 \\
128 & $1.75 \mathrm{e}-04$ & 1.50 & $2.22 \mathrm{e}-04$ & 1.47 & $8.27 \mathrm{e}-04$ & 1.12 \\
256 & $5.64 \mathrm{e}-05$ & 1.63 & $6.76 \mathrm{e}-05$ & 1.71 & $3.37 \mathrm{e}-04$ & 1.30 \\
\hline
\end{tabular}

Table 1: Position error norms and convergence rates for the high-density sphere XTransport test case. 


\begin{tabular}{cccccccc}
\hline \multicolumn{7}{c}{ XYZ-Transport position error norms } \\
\hline Grid & $\|\operatorname{Err}\|_{1}$ & $q_{1}$ & $\|$ Err $\|_{2}$ & $q_{2}$ & $\|$ Err $\|_{\infty}$ & $q_{\infty}$ \\
\hline 32 & $4.55 \mathrm{e}-03$ & - & $5.58 \mathrm{e}-03$ & - & $1.39 \mathrm{e}-02$ & - \\
64 & $1.21 \mathrm{e}-03$ & 1.91 & $1.54 \mathrm{e}-03$ & 1.86 & $4.81 \mathrm{e}-03$ & 1.53 \\
128 & $3.32 \mathrm{e}-04$ & 1.87 & $4.19 \mathrm{e}-04$ & 1.88 & $1.68 \mathrm{e}-03$ & 1.51 \\
256 & $1.04 \mathrm{e}-04$ & 1.67 & $1.39 \mathrm{e}-04$ & 1.59 & $6.86 \mathrm{e}-04$ & 1.30 \\
\hline
\end{tabular}

Table 2: Position error norms and convergence rates for the high-density sphere XYZTransport test case.

The following measurements concern the conservation of the normalized kinetic energy $K$ (Eq. (37)) by the different meshes for the two test cases, plotted in respectively Fig. 13 and Fig. 14.

Both graphs show a steady value of $K$, with small fluctuations depending on the mesh size. These oscillations are stable in time and decrease strongly with the mesh size, going from $0.01 \%$ to the seventh decimal for the finest mesh: the mesh convergence rate for the oscillation amplitudes is between two and four. The same behaviour is observed in both test cases. A temporal average value $\|E r r\|=|1-K|$ has been computed in order to perform a comparison with the theoretical unity value, the results plotted in Fig. 15 and resumed in Table 3 as well as the relative convergence rates. 


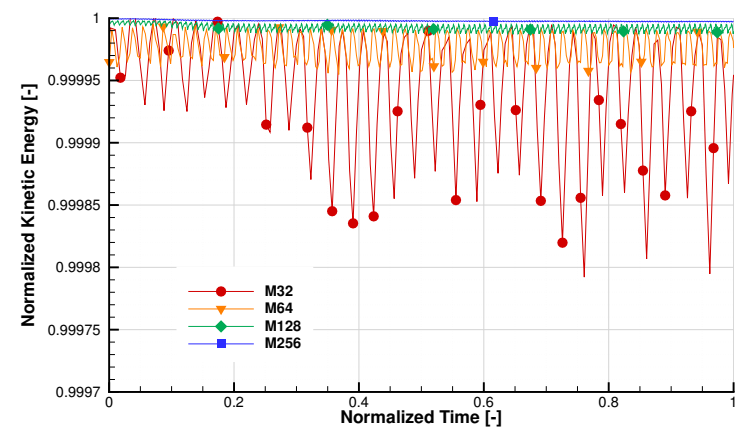

Figure 13: Normalized kinetic energy for the X-transport case, four meshes.

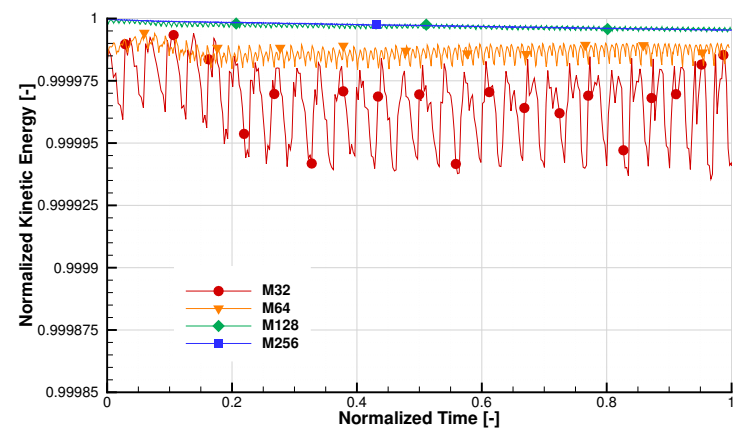

Figure 14: Normalized kinetic energy for the XYZ-transport case, four meshes. 


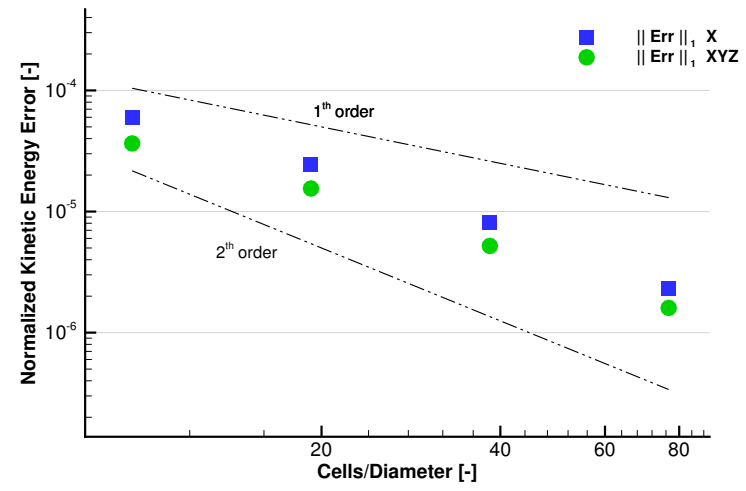

Figure 15: Normalized kinetic energy errors of the high-density fluid sphere after a full flow-through for the transport in X-direction and XYZ-direction. The errors are compared to first and second-order mesh convergences.

\begin{tabular}{ccccc}
\hline & \multicolumn{2}{c}{ X-Transport } & \multicolumn{2}{c}{ XYZ-Transport } \\
\hline Grid & $\|$ Err $\|$ & $q$ & $\|$ Err $\|$ & $q$ \\
\hline 32 & $5.95 \mathrm{e}-05$ & - & $3.65 \mathrm{e}-05$ & - \\
64 & $2.43 \mathrm{e}-05$ & 1.29 & $1.55 \mathrm{e}-05$ & 1.24 \\
128 & $8.10 \mathrm{e}-06$ & 1.58 & $5.20 \mathrm{e}-06$ & 1.58 \\
256 & $2.30 \mathrm{e}-06$ & 1.82 & $1.60 \mathrm{e}-06$ & 1.70 \\
\hline
\end{tabular}

Table 3: Normalized Kinetic energy errors and convergence rates for the high-density fluid sphere in the X-advection and the XYZ-advection test cases.

The results show a very good conservation of the global kinetic energy, in agreement with the conservation of the spherical shape as well as low positioning errors after the full flow-through. The convergence rates are again between first and second order, showing a very good improvements with the mesh refinement. It has to be pointed out that even the coarsest meshes exhibit a good behaviour, so that in low-resolution scenarios the code is expected to track reasonably well 
very small structures without drastic mass losses. These results suggest that the proposed numerical method is able to solve almost arbitrary high density ratio flows ${ }^{3}$ with good accuracy and mesh convergence behaviour as well as a robust behaviour for low-resolved liquid structures. The splitting effects are visible but they do not impact significantly the global performances of the code.

\subsection{Static water drop in a uniform airflow}

Here is reported a test case, already presented in Xiao et al. [31], that represents a good and fast benchmark to demonstrate the advantages of the new convective algorithm (CMOM) with respect to the classical Coupled LevelSet/Volume of Fluid/WENO (CLSVOF) method. A drop of water, with a radius $R=1.55 \mathrm{~mm}$, is placed in a uniform air flow, $U_{g}=15.7 \mathrm{~ms}^{-1}$. The density and the dynamic viscosity of the air and of the water are: $\rho_{g}=1.272 \mathrm{~kg} \mathrm{~m}^{-3}$, $\mu_{g}=1.86 \times 10^{-5} \mathrm{~kg}(\mathrm{~ms})^{-1} ; \rho_{l}=1000 \mathrm{~kg} \mathrm{~m}^{-3}, \mu_{l}=0.892 \times 10^{-3} \mathrm{~kg}(\mathrm{~ms})^{-1}$. The drop is located at the center of a cubical domain of length $7.75 \mathrm{~mm}$ and the considered grid is $64^{3}$. In this simulation the surface tension coefficient is purposely set equal to zero to avoid the related pressure jump across the interface.

The pressure field referred to first time instants of the simulations, $t=$ $4 \times 10^{-5} s$, is analysed. At this time, no wake region has yet formed behind the drop, see Fig. 16, the drop is still spherical and its center should not have moved. The pressure field, in these conditions, is similar to that produced by a gas flow around a solid sphere: the pressure increases in the gas phase up to its maximum value at the stagnation point, where $\mathbf{V}=0$, located at the drop interface (as shown by Fig. 16). Inside the drop, the pressure should approach the linear solution, since the pressure Poisson equation has a negligible source term due to the small velocity in the liquid phase. In consequence, it should vary smoothly between the higher values at the upstream and downstream stagnation points.

\footnotetext{
${ }^{3}$ Subsequent numerical tests whit density ratios up to $10^{9}$ have been successfully carried out.
} 
Moreover, the value of the pressure at the drop center should be similar to the pressure imposed as outflow condition on the right side of the domain. Fig. 17 shows the pressure field on the $x y$ plane at $z=0$ obtained with the CMOM, Fig. 17a, and the classical CLSVOF method, Fig. 17b. In both cases the highest pressure is reached at the front stagnation point, but the result obtained with the CLSVOF presents some non-physical pressure jumps close to the interface and a strong depression inside the drop. Such pressure jumps are due to the momentum errors at the interface caused by the high density ratio that is not taken into account by the classical discretization of convective terms. Because of these errors, the interface of the drop is perturbed and appears irregular. Fig. 18a shows the pressure distribution in the $x$-direction at $y=0$ and $z=0$. With the new algorithm, represented by the red line with circles, a smooth pressure distribution is obtained even near the interface. The CLSVOF result, the blue line with diamonds, instead, not only presents a non smooth pressure close to the interface, see the insight of Fig. 18a, but is not able to correctly represent the stagnation point: the velocity obtained at this location with this scheme is one order of magnitude greater than the one evaluated with the new algorithm, see the insight of Fig. 18b. 


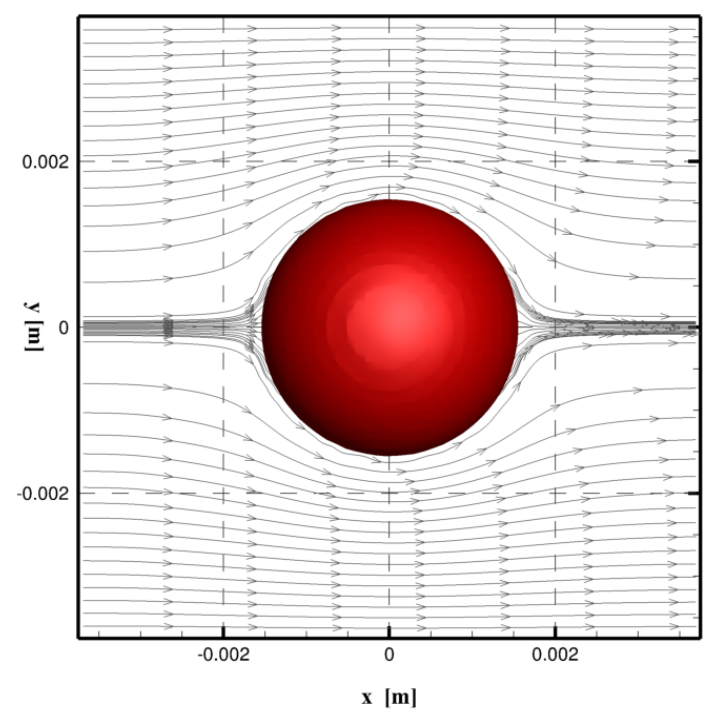

Figure 16: Streamlines around a static water drop in uniform air flow at early times.

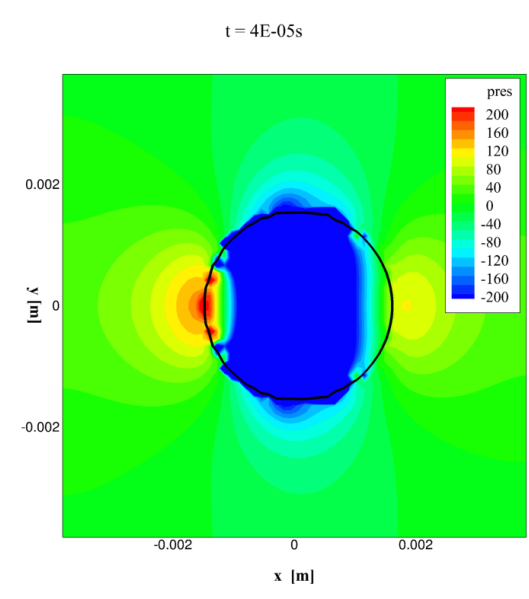

(a) CLSVOF-WENO

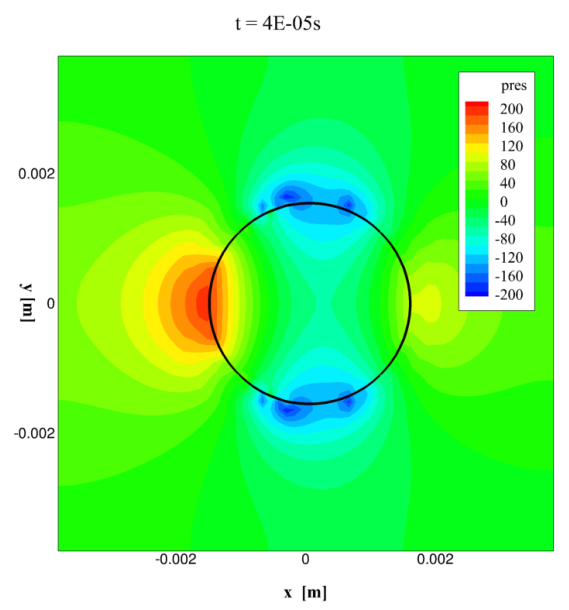

(b) $\mathrm{CMOM}$

Figure 17: Pressure distribution on a plane through the drop mid-section obtained with (a) the classical CLSVOF-WENO algorithm, (b) the CMOM method. 


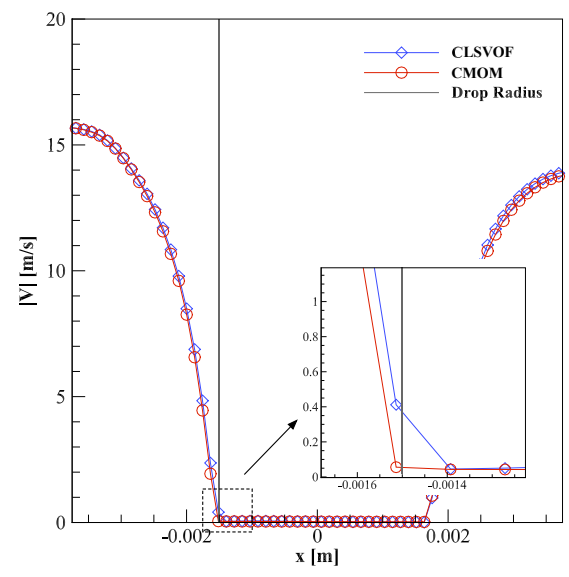

(a) Velocity

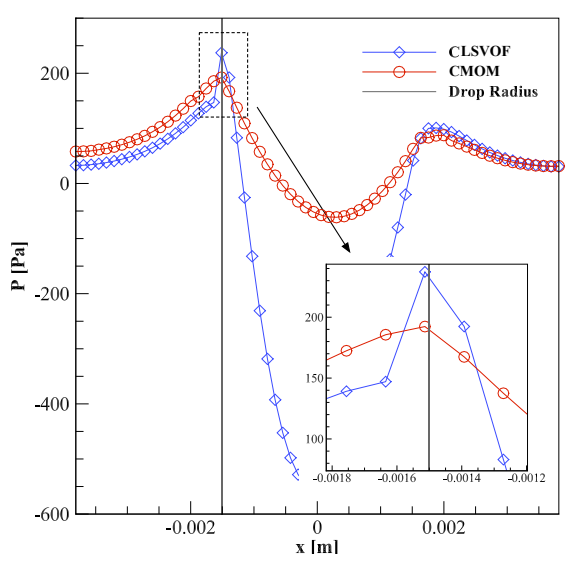

(b) Pressure

Figure 18: (a) Velocity and (b) pressure distribution in the $x$-direction at $y=0$ and $z=0$. The insights report the distributions around the upstream stagnation point.

\subsection{Rising bubble}

This test case involves a gaseous bubble rising by the action of buoyancy forces in a column of liquid. In this test, all the physical terms in Eq. (2) are considered and relevant for the simulation. The proposed convection scheme is used for the advection terms. An experimental investigation of various rising bubble configurations has been proposed by Bhaga and Weber [32]. In this work, air bubbles are formed in a large column containing an aqueous sugar solution of varying concentration. The bubbles are classified by means of three dimensionless groups:

$$
\begin{aligned}
\text { Reynolds number, } \quad R & =\frac{\rho U d}{\mu} \\
\text { Eötvös number, } \quad E & =\frac{\rho g d^{2}}{\sigma} \\
\text { Morton number, } \quad M & =\frac{g \mu^{4}}{\rho \sigma^{3}}
\end{aligned}
$$

being $\rho$ the liquid density, $U$ the terminal rise velocity, $d$ the bubble diameter, $\mu$ the liquid viscosity, $\sigma$ the surface tension coefficient ans $g$ gravity. Among the available results, the authors Bhaga and Weber [32] propose photo-shots of 
the bubbles as well as a correlation approximating their terminal rising velocity. At fixed Eötvös, variations of the Morton and Reynolds numbers were achieved by modifying the sugar-water liquid viscosity. Simulations of two of these proposed experiences are proposed by Owkes and Desjardins [10] by a mass and momentum preserving un-split method based on the Rudman fine-mesh approach. The currently proposed single-grid CMOM method is tested against both experiences and numerical simulations. Two test cases are considered, the respective characteristics presented in Table 4 .

\begin{tabular}{cccc}
\hline Case & Re & Eo & Mo \\
\hline 1 & 2.47 & 116 & 848 \\
2 & 7.16 & 116 & 41.1 \\
\hline
\end{tabular}

Table 4: Non-dimensional numbers for the rising bubble test cases.

For each test cases, two mesh converging simulations are performed. The computational domain consists of a rectangular tank of size $L=[8 D \times 16 D \times 8 D]$, being $D$ the bubble initial diameter. The mesh size are respectively $M 128=$ $N_{x} \times N_{y} \times N_{z}=[128 \times 256 \times 128]$ and $M 256=N_{x} \times N_{y} \times N_{z}=[256 \times 512 \times 256]$. Slip conditions are applied on all of the domain boundaries. The simulations are performed until stabilization of the bubble shape and rise velocity. The numerical terminal velocities are compared, as suggested by Owkes and Desjardins [10], to the correlation proposed by Angelino Angelino [33], based on the same experience :

$$
U=K V^{m}
$$

The coefficients $K$ and $m$ are obtained by least-square interpolation of experimental data, leading to:

$$
\begin{gathered}
K=25 /\left(1+0.33 M^{0.29}\right) \\
m=0.167 /\left(1+0.34 M^{0.24}\right)
\end{gathered}
$$


A comparison is made with the two-grids approach of Owkes and Desjardins Owkes and Desjardins [10] as well.

(a)

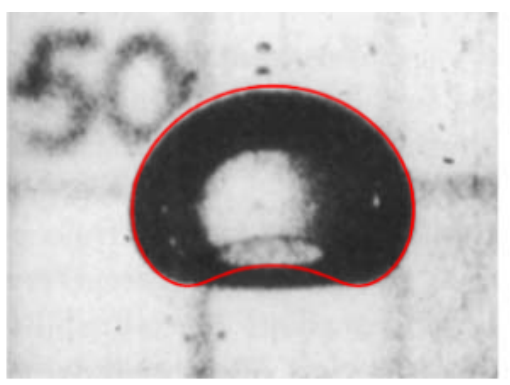

(a) $\mathrm{Mo}=848$

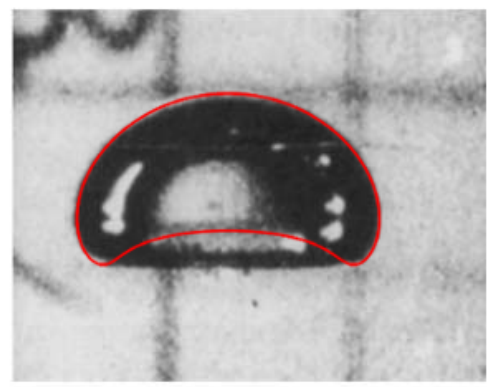

(b) $\mathrm{Mo}=041$

Figure 19: Comparison of the bubble shapes between the proposed numerical scheme and the experimental visualizations of Bhaga and Weber Bhaga and Weber [32]. (a) Case 1, Mo $=848, t \approx 0.8 s$ in the simulation (b) Case 2, Mo $=41, t \approx 0.3 s$ in the simulation.

Fig. 19 shows a snapshot of the bubble profile once the steady-state solution is well established, superposed to the experimental visualizations from Bhaga and Weber [32]. The bubble profiles have been obtained by 2D slices cutting through the bubble center, thus showing the inner cavity. Both shapes compare very well with the experimental results. Similar qualitative results have been obtained in Owkes and Desjardins [10]. 20 presents the time-histories of the mean rise velocity $V_{\text {rise }}$. Its value has been obtained by integrating the vertical component $v$ of the velocity inside the bubble of volume $V_{b u b}$ at each time-step by equation (49):

$$
V_{\text {rise }}(t)=\frac{1}{V_{b u b}} \sum_{i} v_{i}(t) C_{i}(t) \Delta x^{3}
$$

The graphs show the rise velocities attaining their steady-state values for both mesh sizes, the higher Morton case (Case 1) showing a longer characteristic settling time. For the Case 1, the velocity shows a steady increase in time. The coarser mesh achieve a steady state solution at around $t \approx 0.6$, while for the finer mesh it takes a little longer, $t \approx 0.8$. Numerical results are detailed in 
Table 5 .

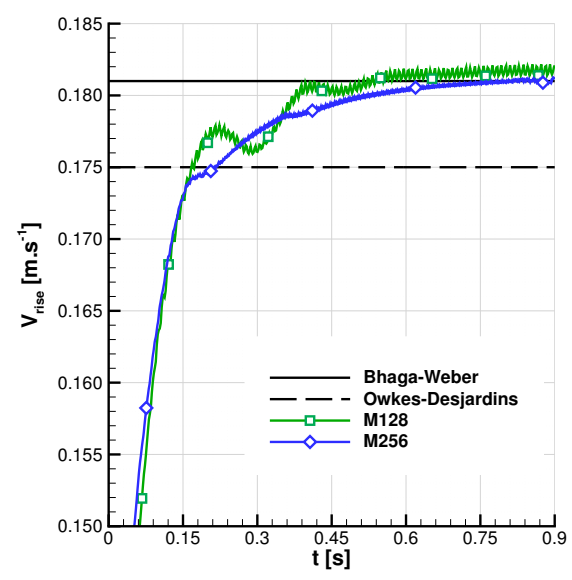

(a) $\mathrm{Mo}=848$

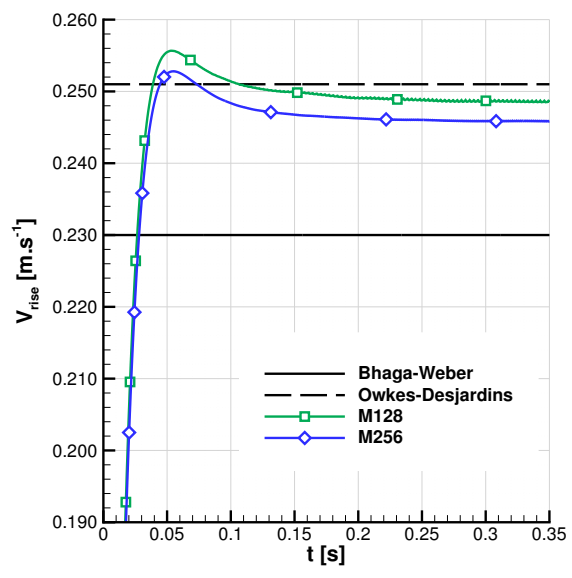

(b) $\mathrm{Mo}=041$

Figure 20: Time history of the bubble rise velocity by the proposed numerical scheme with M128 and M256 meshes, comparison with terminal velocities from the experimental measurements of Bhaga and Weber Bhaga and Weber [32] and the simulations results of Owkes and Desjardins Owkes and Desjardins [10]. (a) Case 1, Mo $=848$ (b) Case $2, \mathrm{Mo}=41$.

\begin{tabular}{|c|c|c|c|}
\hline \multirow[t]{2}{*}{ Case } & \multicolumn{3}{|c|}{ Rise Velocity $\left[m . s^{-1}\right]$} \\
\hline & Proposed scheme & Owkes \& Desjardins & Bhaga \& Weber \\
\hline \multirow[t]{2}{*}{1} & 0.182 (M128) & 0.175 & 0.181 \\
\hline & 0.181 (M256) & & \\
\hline \multirow[t]{2}{*}{2} & 0.249 (M128) & 0.251 & 0.230 \\
\hline & 0.246 (M256) & & \\
\hline
\end{tabular}

Table 5: Terminal velocities of the rising bubbles, Case 1 and 2, comparison with literature results (Owkes and Desjardins [10] and Bhaga and Weber [32]) for the two proposed meshes. Values rounded to the third decimal.

The experimental results are very well predicted by the present method. The 
resulting velocity of Case 1 achieve less than $0.5 \%$ relative difference compared to the correlation (46), even with the coarser mesh. Results of Case 2 show a global difference of about $7 \div 5 \%$ between numerics and experiences depending on the mesh size. The presented results seem to agree with those of Owkes and Desjardins [10], obtained with a different numerical method, even slightly improving the velocity values.

Overall, the presented two-phase flow solver has proven capable to correctly reproduce low Reynolds numbers flows dominated by viscous and capillary effects. The next step is to validate the new CMOM method against a more severe atomization test case, where important momentum exchanges occur between the two liquid phases.

\subsection{Liquid jet in cross-flow}

The transverse injection of a liquid jet in a high speed gaseous cross-flow (LJICF) is applied in many engineering applications, like the air breathing propulsion systems, since this technique allows to achieve good liquid/gas mixing. For this reason such flow has been extensively studied both from the experimental and numerical point of view. Although 3D unsteady simulations of a jet in cross-flow have made encouraging progress in last years, they still represent a very challenging problem in particular for the high liquid/gas density ratio characterizing the flow. To reduce the numerical errors, indeed, many simulations have been performed only at low density ratio, as for example in Behzad et al. [34], Andreini et al. [35] or Herrmann [36]. High density ratio simulations can however be found in Li and Soteriou [37] or Xiao et al. [38], where a particular technique based on velocity extensions at the interface location allowed a robust simulation of the assisted air-water atomization at ambient pressure. 


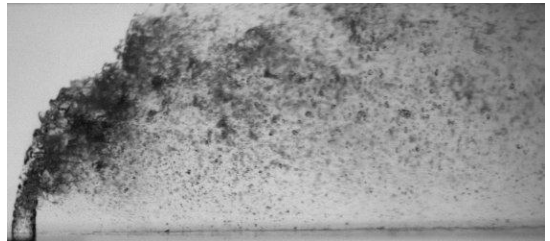

(a) $q \approx 7.5$

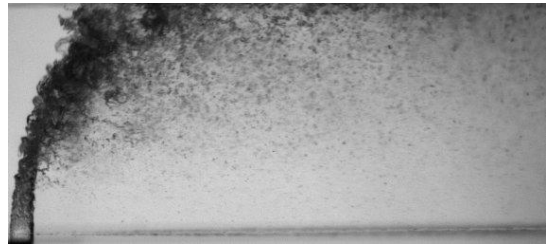

(b) $q \approx 11.8$

Figure 21: Two instantaneous visualizations of the liquid jet atomization from ONERA experiences of Bodoc et al. [39] and Bodoc et al. [40].

Several dimensionless parameters can be chosen to classify the jet atomization behaviour. In particular, the momentum flux ratio (50) and the cross-flow Weber number (51):

$$
\begin{aligned}
q & =\frac{\rho_{l} u_{l}^{2}}{\rho_{g} u_{g}^{2}} \\
W e & =\frac{\rho_{g} u_{g}^{2} d_{j}}{\sigma}
\end{aligned}
$$

where $u_{k}$ is the average inflow velocity, $\rho_{k}$ the gas density, $\sigma$ the surface tension coefficient for $k=l, g$ and $d_{j}$ the jet diameter. Experimental observations of non-turbulent jets in high cross-flow $W e$, see Mazallon et al. [41], Mazallon et al. [41] and Sallam et al. [42], have shown that $W e$ has mainly an impact on the jet breakup, while $q$ influences mostly the jet shape and its penetration, without any significant effect on the breakup process. From Wu et al. [43], Broumand and Birouk [44] and No [45], it is clear that in the liquid jet trajectory the role of the momentum $q$ is widely accepted as the predominant one. It is straigtforward to understand that the bending of the main jet body is linked to the momentum transferred between the high speed gas and the high density liquid column. However, still important discrepancies exist between literature correlations. For this reason, the present work attempts a comparison with the particular experience of Bodoc et al. [39], where the liquid jet trajectories have been evaluated for several values of $q$, obtained by varying the liquid injection velocity. The aim of this test is not to fully investigate the atomization up to the droplet formation, but rather to focus on the capability of the proposed 
scheme to reproduce the complex momentum exchange between gas and liquid in a high density ratio $\left(10^{3}\right)$ and strong shearing $\left(u_{g} / u_{l} \approx 60\right)$ configuration.

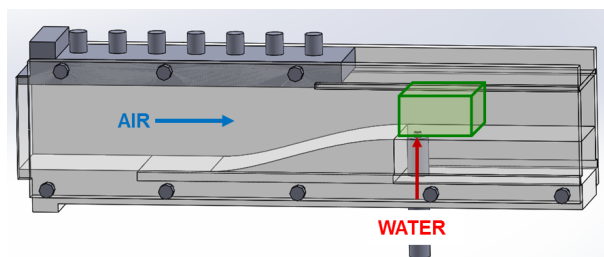

(a)

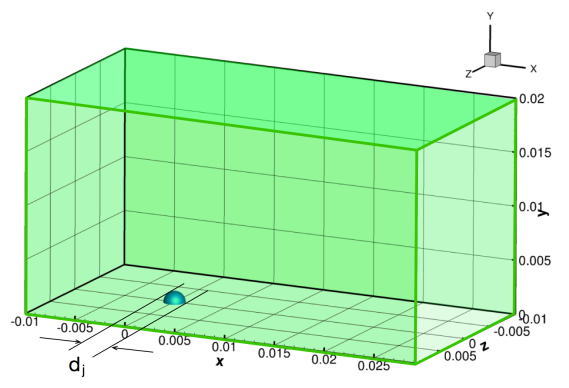

(b)

Figure 22: (a) Schematic representation of the experimental test-bed ; (b) the green box is the considered computational domain.

Fig. 22a shows a schematic representation of the experimental test-bed. The green area represents the considered computational domain: it consists of a rectangular channel in which the liquid jet is injected from a circular orifice on the lower wall, see Fig. 22b. The domain dimensions are $\left[L_{x} \times L_{y} \times L_{z}\right]=[40 \times$ $20 \times 20] \mathrm{mm}$, representing the real height (but not the width) of the channel. The exit diameter of the liquid nozzle is $d_{j}=2 \mathrm{~mm}$ and it is located at $10 \mathrm{~mm}$ from the left boundary where the air inflow is imposed. Note that, in the test-bed, the gas flows through a converging section which ensures a laminar boundary layer development. To sum up, the chosen boundary conditions of the numerical domain are: inflow for the gas stream at $x_{\min }$, slip at $z_{\min }$ and $z_{\max }$, no-slip at $y_{\max }$ and $y_{\min }$ boundaries, with the exception of the jet inflow, and free-outflow at $x_{\max }$. The Cartesian grid has been set to $\left[N_{x} \times N_{y} \times N_{z}\right]=[1024 \times 512 \times 512]$ in order to obtain a resolution of $\Delta x=4 \times 10^{-5} \mathrm{~m}$, a cell size appropriate to resolve the primary breakup of the jet. The considered fluid properties (air and water) are presented in Table 6 . 


\begin{tabular}{r|cl}
\hline Gas density & $\rho_{g}=1.225$ & {$\left[\mathrm{~kg} \mathrm{~m}^{-3}\right]$} \\
Liquid density & $\rho_{l}=1000$ & {$\left[\mathrm{~kg} \mathrm{~m}^{-3}\right]$} \\
Gas viscosity & $\mu_{g}=1.78 \times 10^{-5}$ & {$\left[\mathrm{~kg}(\mathrm{~ms})^{-1}\right]$} \\
Liquid viscosity & $\mu_{l}=1.0 \times 10^{-3}$ & {$\left[\mathrm{~kg}^{\left.\mathrm{m} \mathrm{s})^{-1}\right]}\right.$} \\
Surface tension & $\sigma=7.2 \times 10^{-2}$ & {$[\mathrm{Nm}]$} \\
\hline
\end{tabular}

Table 6: Gas and liquid dimensional properties.

A non-turbulent uniform-profile inflow has been used for the gas, as the canalization ramp is supposed to minimize turbulence and boundary layers. For the liquid, a more accurate inlet profile has been used to reproduce the water injector. A RANS simulation of the injector has been performed in the work of Thuillet [46]: the average injection velocity profile resulting from the RANS has been injected in the DNS inflow condition. No velocity fluctuations have been injected in the liquid flow, a point which should be probably addressed for future simulations.

The trajectory of the jet heavily depending on this mechanism, simulations for two air stream velocities, giving two values of $q$, have been performed, see Table 7 .

\begin{tabular}{ccc}
\hline Case & q & We \\
\hline 1 & 7.5 & 144 \\
2 & 11.8 & 92 \\
\hline
\end{tabular}

Table 7: Cross-flow Weber and momentum flux ratio numbers.

Fig. 23 illustrates the evolution of the jet interface for the two simulations. It is possible to notice many characteristics of a multimode/shear breakup described in Mazallon et al. [41] and Sallam et al. [42], among others. 


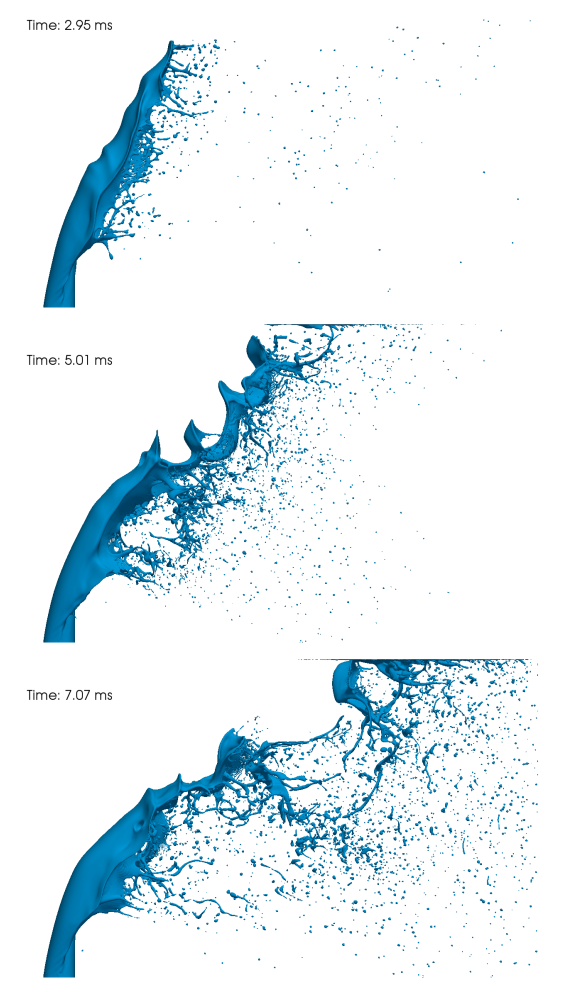

(a) $q=7.5$

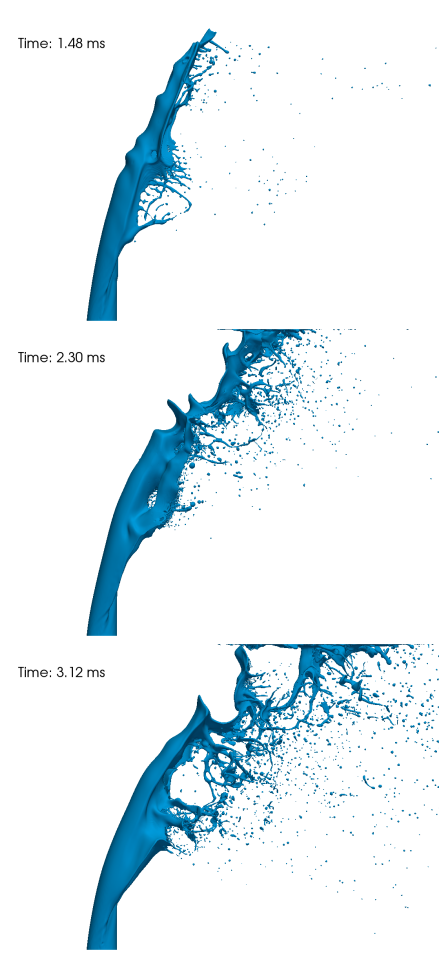

(b) $q=11.8$

Figure 23: Evolution of the cross-flow jet atomization, for the two values of $q=$ $\rho_{l} u_{l}^{2} / \rho_{g} u_{g}^{2}$, side view, three instants. The air flow is coming from the left side.

The breakup mechanism begins with the jet deflection in the cross-flow direction, due to the aerodynamic force. Regular wavelike disturbances appear on the windward surface of the deformed liquid jet. The propagation of these long waves induces the liquid column breakup by means of the separation of liquid packets undergoing further rupture. In a pure shear breakup regime the instabilities wavelength are of the order of $\lambda \approx 0.1 d_{j}$ Sallam et al. [42]; for lower We, or bag/multi-modal breakup the wavelength approaches the diameter of the jet, $\lambda \approx d_{j}$. In the presented simulations, those larger wavelength are clearly visible. Smaller wavelengths can be hinted but do not seem to be relevant for the atomization of the jet. It is possible that the absence of injected liquid turbulence 
and the confinement of the jet do not allow their development. However, the instabilities wavelength do not influence the global jet trajectory. Conforming to the numerical simulations presented in Xiao et al. [38] and Behzad et al. [34], at the considered $W e$, formation of ligaments is due to a pinching mechanism. Indeed, in the initial phase of the liquid-gas interaction, because of the high $W e$, liquid sheets are ejected in the span-wise direction, along the periphery of the jet. These sheets elongate into ligaments undergoing further Rayleigh-Plateau breakup.

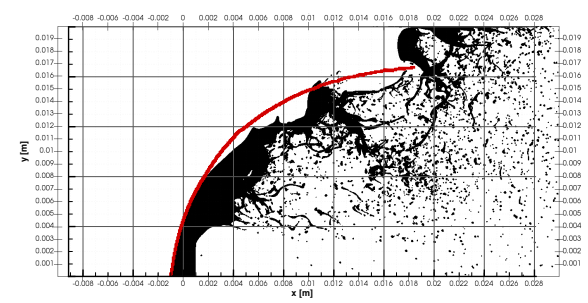

(a) $q=7.5$, instantaneous profile.

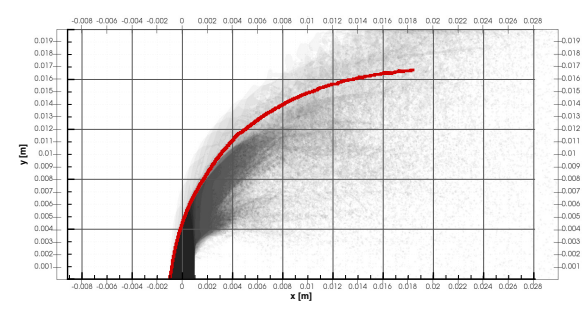

(c) $q=7.5$, averaged profile.

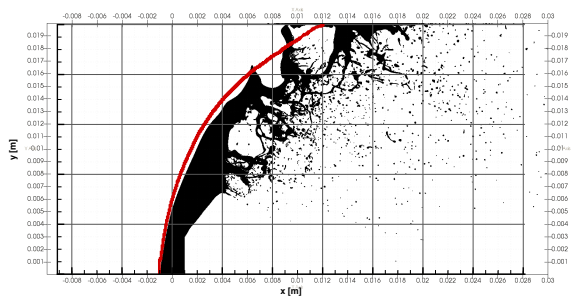

(b) $q=11.8$, instantaneous profile.

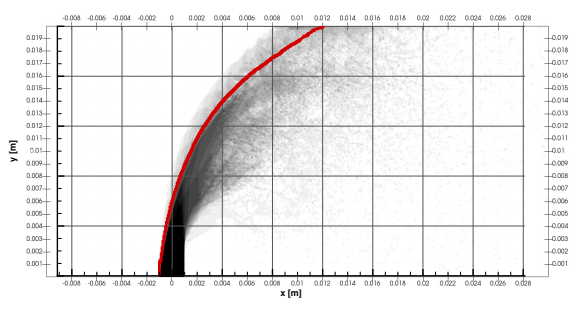

(d) $q=11.8$, averaged profile.

Figure 24: Cross flow liquid jet shapes for both values of $q$, compared to the experimental trajectories from Bodoc et al. [40](red continuous lines). (a) and (b), instantaneous profiles; (c) and (d), averaged intensity profiles.

The difference between the two regimes $q=7.5$ and $q=11.8$ is visible in the evolution of Fig.s 23a and 23b. The lesser gas momentum of the $q=11.8$ Case 2 allows the jet to stand more upright and to directly impact the upper wall. The Case 1 jet, $q=7.5$, appears more bent and breaks-up before impacting the wall : 
large liquid structures detaches from the body before undergoing pulverisation. The behaviour of the jet is in good agreement with the visualizations depicted in Fig. 21. Fig. 24 compares the predicted liquid jet shape with the ONERA experimental observation realized in Bodoc et al. [39] and Bodoc et al. [40]. Fig.s 24a and 24b depict the instantaneous side view of the jets, while Fig.s 24c and $24 \mathrm{~d}$ show a time-averaged intensity contour of the liquid presence. Even with a low temporal convergence, the shape of the jet is in agreement with the correlations

The presented simulation demonstrates the capabilities of the new consistent mass-momentum transport to reproduce experimental atomization conditions characterized by high density ratio (equal to $10^{3}$ ) and high momentum flux ratio, that were beyond the reach of the previous velocity-based advection scheme.

\section{Remarks on computational efficiency}

The proposed algorithm uses some ideas already presented original works of Ghods and Herrmann [9] and Rudman [8]. From the latter, in particular, the idea of a sub-cell resolution for the volume fraction is employed, as well as the idea of evaluating the momentum-flux density according to the reconstructed volume fraction. However, it has been shown by the presented test cases that an expensive finer grid as advised by Rudman is not needed to achieve consistency.

In order to asses the computational efficiency of the proposed method, some comparative run-time measurements of DYJEAT have been performed on the most computationally intensive test case $\S 4.1$. In this test case only the advective and pressure terms are solved. No viscosity, surface tension nor source terms are considered. A fixed number of time-steps have been set, on an fixed mesh and on a single core. The runs have been performed with the Level-Set-WENO, the CLSVOF-WENO (the non-consistent velocity treatment) and the CLSVOFCMOM approach. A CLSVOF-WENO run on a twice finer mesh $\Delta x / 2$ has been performed as well. The pressure solver iterations number have been fixed as well to an averaged value, so that its contribution is independent of the advection 
algorithm.

Timers have been extracted by using the MPI_Wtime Fortran MPI function. The total execution time has been extracted, as well as the interface tracking, the momentum update and the pressure solver run-times. As the absolute computational times may strongly depend on architecture, compiler and/or code optimization, only relative times are presented.

Table 8 shows the measured times, normalized by the absolute lowest value (the Level-Set Interface tracking time on the $\Delta x$ mesh).

\begin{tabular}{r|c|ccc}
\hline Algorithm & Mesh & TOT & IT & MU \\
\hline Level-Set (LS) & $\Delta x$ & 26.9 & $\mathbf{1 . 0}$ & 1.3 \\
CLSVOF-WENO & $\Delta x$ & 27.5 & 2.4 & 1.3 \\
CLSVOF-CMOM & $\Delta x$ & 28.9 & 2.4 & 2.5 \\
\hline CLSVOF-WENO & $\Delta x / 2$ & 285.9 & 14.1 & 8.7 \\
\hline
\end{tabular}

Table 8: Normalized run-time measurements of $\S 4.1$ test case, for the different algorithms. TOT : total time; IT: interface tracking; MU: momentum update. Normalization by the lowest absolute value (LS-IT).

Several conclusions can be drawn from these results :

- The LS algorithm is the least expensive in DYJEAT.

- The coupling with the VOF in DYJEAT shows a $150 \%$ increment in the interface tracking compared to the LS. The same WENO scheme is used for the velocity update.

- Switching to a CMOM momentum advection almost doubles the velocity update time, an expected result coming from the added temporary continuity equations.

- Computing the CLSVOF operations on a twice finer mesh gives, not surprisingly, a factor 6 on the interface tracking, and 7 on the velocity update. 
Indeed, $2^{3}=8$ times more operations must be theoretically performed for each equation.

This preliminary analysis suggests that the computational excess of the CLSVOF-CMOM would be much lower than using a Rudman-type method using a twice as fine grid. The total CPU time excess of CLSVOF-CMOM compared to CLSVOF-WENO computations amounts to $5 \% \div 10 \%$. An important benefit in terms of RAM memory is also expected: in the proposed scheme there are some sub-grid quantities to be computed, but never stored. Defining VOFrelated equations on the finer mesh would imply storing several variables on the finer grid. Code optimization considerations should be taken into account at this point, however.

\section{Conclusions}

In this paper, an efficient method to achieve a consistent momentum-preserving transport for two-phase flows simulation on staggered grids has been presented. Starting from a standard Coupled Level-Set/VOF directionally split approach, the main ideas behind this method are: (i) to recast the momentum equation in a conservative form; (ii) to solve auxiliary continuity equations coupled to the momentum equations in the velocity control volumes; (iii) to evaluate consistent mass and momentum fluxes by means of the PLIC reconstruction.

The continuity and momentum fluxes are evaluated by considering the equivalence between mass and VOF as in Rudman [8]. The inconsistency related to the staggered arrangements is dealt with by temporary continuity equations, solved on the face-centered velocity control volumes. These allow recovering a consistent updated velocity, which avoids the development of instabilities related to momentum transfer and grants good kinetic energy conservation properties.

The Rudman's resolution of the continuity and momentum equations on a twice finer sub-grid is not needed, thus avoiding the significant additional computational cost. Compared to un-split advection schemes, the proposed scheme retains the simplicity of the directional PLIC fluxes computation, without the 
complex reconstruction of the three-dimensional streak-tubes. Mass conservation is satisfied for all practical purpose, even though machine error conservation is not systematically achieved, thanks to the VOF contribution.

Different test cases show a significant improvement in both stability and accuracy of this new method, even at extreme density ratios of $10^{6}$. In particular, the liquid jet in cross-flow test case can be considered as a difficult atomization test case: the new method can handle both high density and shearing ratios, whereas the classical velocity-based advection schemes for momentum fail. The liquid jet trajectory, which depends mainly on the ratio of liquid-to-gas momentum flux, is successfully validated against ONERA experimental data. Even

very small droplets can be tracked in a strong shearing flow, which is one of the goals of atomization DNS.

Finally, the CPU time increase induced by the new method is less than $10 \%$ higher than the standard CLSVOF-WENO scheme.

\section{Acknowledgment}

The present study has been granted by the Foundation STAE-RTRA-research program SIMACO3FI. The authors are grateful to STAE-RTRA Foundation for having funded this study and especially the post-doc of Dr. A. Orazzo.

This work was granted access to the HPC resources of CINES under the allocation 2017 - A0032B06115 made by GENCI, and the HPC resources of CALMIP under the allocation 2018 - Project 18043.

We would like to thank T. Menard and A. Berlemont for the many insightful discussions on the topic.

[1] A. J. Chorin, Numerical solution of the navier-stokes equations, Mathematics of Computation 22 (1968) 745-762.

[2] G. Tryggvason, R. Scardovelli, S. Zaleski, Direct Numerical Simulation of Gas-Liquid Multiphase Flows, Cambridge University Press, 2011. 
[3] D. Q. Nguyen, R. P. Fedkiw, M. Kang, A boundary condition capturing method for incompressible flame discontinuities, Journal of Computational Physics 172 (2001) $71-98$.

[4] M. Sussman, K. Smith, M. Hussaini, M. Ohta, R. Zhi-Wei, A sharp interface method for incompressible two-phase flows, Journal of Computational Physics 221 (2007) $469-505$.

[5] F. Xiao, Large Eddy Simulation of Liquid Jet Primary Breakup. PhD Thesis., Ph.D. thesis, Loughborough Univ, 2012.

[6] M. Raessi, H. Pitsch, Consistent mass and momentum transport for simulating incompressible interfacial flows with large density ratios using the level set method, Computers and Fluids 63 (2012) 70 - 81.

[7] I. Park, H. Cho, H. Yoon, J. Jeong, Numerical effects of the semiconservative form of momentum equations for multi-dimensional two-phase flows, Nuclear Engineering and Design 239 (2009) 2365 - 2371.

[8] M. Rudman, A volume-tracking method for incompressible multifluid flows with large density variations, International Journal for Numerical Methods in Fluids 28 (1998) 357-378.

[9] S. Ghods, M. Herrmann, A consistent rescaled momentum transport method for simulating large density ratio incompressible multiphase flows using level set methods, Physica Scripta 88 (2013).

[10] M. Owkes, O. Desjardins, A mass and momentum conserving unsplit semilagrangian framework for simulating multiphase flows, Journal of Computational Physics 332 (2017) $21-46$.

[11] M. Bussmann, D. Kothe, J. Sicilian, Modelling high density ratio incompressible interfacial flows, in: Proceedings of Engineering Division Summer Meeting, ASME Fluids, Montreal, Canada, 2002, pp. 1-7. 
[12] G. Vaudor, T. Ménard, W. Aniszewski, M. Doring, A. Berlemont, A consistent mass and momentum flux computation method for two phase flows. application to atomization process, Computers and Fluids 152 (2017) 204 $-216$.

[13] V. LeChenadec, H. Pitsch, A monotonicity preserving conservative sharp interface flow solver for high density ratio two-phase flows, Journal of Computational Physics 249 (2013) 185 - 203.

[14] R. Scardovelli, S. Zaleski, Direct numerical simulation of free-surface and interfacial flow, Annual Review of Fluid Mechanics 31 (1999) 567-603.

[15] J. A. Sethian, Level Set methods and Fast Marching methods, Cambridge University Press, 1999.

[16] O. Desjardins, V. Moureau, Methods for multiphase flows with high density ratio, in: Proceedings of the Summer Program, Center of turbulence research, Stanford, 2010, pp. 313-322.

[17] R. P. Fedkiw, T. Aslam, B. Merriman, S. Osher, A non-oscillatory eulerian approach to interfaces in multimaterial flows (the ghost fluid method), Journal of Computational Physics 152 (1999) 457 - 492.

[18] J. K. Patel, G. Natarajan, A novel consistent and well-balanced algorithm for simulations of multiphase flows on unstructured grids, Journal of Computational Physics 350 (2017) $207-236$.

[19] D. Fuster, T. Arrufat, M. Crialesi-Esposito, Y. Ling, L. Malan, S. Pal, R. Scardovelli, G. Tryggvason, S. Zalesk, A momentum-conserving, consistent, volume-of-fluid method for incompressible flow on staggered grids, arXiv:1811.12327 [physics.comp-ph] (2020).

[20] M. Sussman, E. G. Puckett, A coupled level set and volume-of-fluid method for computing 3d and axisymmetric incompressible two-phase flows, Journal of Computational Physics 162 (2000) $301-337$. 
[21] T. Menard, S. Tanguy, A. Berlemont, Coupling level set/vof/ghost fluid methods: Validation and application to 3d simulation of the primary breakup of a liquid jet, International Journal of Multiphase Flow 33 (2007) 510 -524 .

[22] F. Couderc, Développement d'un code de calcul pour la simulation d'écoulements de fluides non miscibles. Application à la désintégration assistée d'un jet liquide par un courant gazeux. (Development of a numerical code for the simulation of non-miscible fluid flow. Application to the airassisted disintegration), Ph.D. thesis, ISAE, Université de Toulouse, 2007.

[23] D. Zuzio, J. Estivalèzes, An efficient block parallel amr method for two phase interfacial flow simulations, Computers and Fluids 44 (2011) 339 357.

[24] D. Zuzio, J.-L. Estivalèzes, B. DiPierro, An improved multiscale eulerianlagrangian method for simulation of atomization process, Computers and Fluids In Press (2018).

[25] T. Xavier, D. Zuzio, M. Averseng, J.-L. Estivalezes, Toward direct numerical simulation of high speed droplet impact, Meccanica (2019).

[26] J. Lopéz, J. Hernandez, Analytical and geometrical tools for 3d volume of fluid methods in general grids, Journal of Computational Physics 227 (2008) $5939-5948$.

[27] G. Strang, On the Construction and Comparison of Difference Schemes, SIAM Journal on Numerical Analysis 5 (1968) 506-517.

[28] E. G. Puckett, A. S. Almgren, J. B. Bell, D. L. Marcus, W. J. Rider, A high-order projection method for tracking fluid interfaces in variable density incompressible flows, Journal of Computational Physics 130 (1997) 269 282. 
[29] M. Kang, R. P. Fedkiw, X.-D. Liu, A boundary condition capturing method for multiphase incompressible flow, Journal of Scientific Computing 15 (2000) 323-360.

[30] O. Tatebe, The multigrid preconditioned conjugate gradient method, in: Proceedings of the 6th Copper Mountain Conference on Multigrid Methods, volume CP-3224, Copper Mountain, 1993, p. 621.

[31] F. Xiao, M. Dianat, J. McGuirk, A robust interface method for drop formation and breakup simulation at high density ratio using an extrapolated liquid velocity, Computers and Fluids 136 (2016) $402-420$.

[32] D. Bhaga, M. E. Weber, Bubbles in viscous liquids: shapes, wakes and velocities, Journal of Fluid Mechanics 105 (1981) 61-85.

[33] H. Angelino, Hydrodynamique des grosses bulles dans les liquides visqueux, Chemical Engineering Science 21 (1966) 541 - 550.

[34] M. Behzad, N. Ashgriz, B. Karney, Surface breakup of a non-turbulent liquid jet injected into a high pressure gaseous crossflow, International Journal of Multiphase Flow 80 (2016) 100 - 117.

[35] A. Andreini, C. Bianchini, S. Puggelli, F. Demoulin, Development of a turbulent liquid flux model for eulerian-eulerian multiphase flow simulations, International Journal of Multiphase Flow 81 (2016) 88 - 103.

[36] M. Herrmann, Detailed numerical simulations of the primary atomization of a turbulent liquid jet in crossflow, Journal of Engineering for Gas Turbines and Power 132 (2010) 061506-10.

[37] X. Li, M. C. Soteriou, Detailed numerical simulation of liquid jet atomization in crossflow of increasing density, International Journal of Multiphase Flow 104 (2018) 214-232.

[38] F. Xiao, Z. Wang, M. Sun, J. Liang, N. Liu, Large eddy simulation of liquid jet primary breakup in supersonic air crossflow, International Journal of Multiphase Flow 87 (2016) 229 - 240. 
[39] V. Bodoc, A. Desclaux, P. Gajan, F. Simon, G. Illac, Characterization of confined liquid jet injected into oscillating air crossflow, Flow, Turbulence and Combustion (2019).

[40] V. Bodoc, A. Desclaux, P. Gaja, F. Simon, G. Illac, Characterization of confined liquid jet injected into oscillating air crossflow, Flow Turbulence and Combustion Accepted for publication (2018).

[41] J. Mazallon, Z. Dai, G. M. Faeth, Primary breakup of non-turbulent round liquid jets in gas crossflows, Atomization and Sprays 9 (1999) 291-311.

[42] K. A. Sallam, C. Aalburg, G. M. Faeth, Breakup of round nonturbulent liquid jets in gaseous crossflow, AIAA Journal (2004).

[43] P.-K. Wu, K. A. Kirkendall, R. P. Fuller, A. S. Nejad, Breakup processes of liquid jets in subsonic crossflows, Journal of Propulsion and Power (1997).

[44] M. Broumand, M. Birouk, Liquid jet in a subsonic gaseous crossflow: Recent progress and remaining challenges, Progress in Energy and Combustion Science 57 (2016) $1-29$.

[45] S.-Y. No, A review on empirical correlations for jet/spray trajectory of liquid jet in uniform cross flow, International Journal of Spray and Combustion Dynamics 7 (2015) 283-313.

[46] S. Thuillet, Simulation numérique multi-échelle de l'injection d'un jet liquide en présence d'une perturbation acoustique (Multi-scale simulation of a liquid jet in cross-flow under acoustic perturbation), Ph.D. thesis, ISAE, Université de Toulouse, 2018. 